\title{
Article \\ Altered Root Growth, Auxin Metabolism and Distribution in Arabidopsis thaliana Exposed to Salt and Osmotic Stress
}

\author{
Ana Smolko ${ }^{1,+(\mathbb{D})}$, Nataša Bauer ${ }^{2,+} \mathbb{D}$, Iva Pavlović 1,3 ${ }^{\mathbb{D}}$, Aleš Pěnčík ${ }^{3}$, Ondřej Novák ${ }^{3}(\mathbb{D}$ \\ and Branka Salopek-Sondi ${ }^{1, * \mathbb{D}}$
}

1 Department for Molecular Biology, Ruđer Bošković Institute, Bijenička Cesta 54, 10000 Zagreb, Croatia; ana.smolko@irb.hr (A.S.); iva.pavlovic@irb.hr (I.P.)

2 Department for Molecular Biology, Faculty of Science, University of Zagreb, Horvatovac 102, 10000 Zagreb, Croatia; nbauer@biol.pmf.hr

3 Laboratory of Growth Regulators, Faculty of Science of Palacký University \& Institute of Experimental Botany of the Czech Academy of Sciences, Šlechtitelů 27, CZ-783 71 Olomouc, Czech Republic; ales.pencik@upol.cz (A.P.); novako@ueb.cas.cz (O.N.)

* Correspondence: salopek@irb.hr; Tel.: +385-1-4561-143

+ These authors contributed equally to this work.

check for

updates

Citation: Smolko, A.; Bauer, N.; Pavlović, I.; Pěnčík, A.; Novák, O.; Salopek-Sondi, B. Altered Root Growth, Auxin Metabolism and Distribution in Arabidopsis thaliana Exposed to Salt and Osmotic Stress. Int. J. Mol. Sci. 2021, 22, 7993.

https://doi.org/10.3390/

ijms22157993

Academic Editor: Richard R.-C. Wang

Received: 16 May 2021

Accepted: 19 July 2021

Published: 27 July 2021

Publisher's Note: MDPI stays neutral with regard to jurisdictional claims in published maps and institutional affiliations.

Copyright: (c) 2021 by the authors. Licensee MDPI, Basel, Switzerland. This article is an open access article distributed under the terms and conditions of the Creative Commons Attribution (CC BY) license (https:// creativecommons.org/licenses/by/ $4.0 /)$.

\begin{abstract}
Salt and osmotic stress are the main abiotic stress factors affecting plant root growth and architecture. We investigated the effect of salt $(100 \mathrm{mM} \mathrm{NaCl})$ and osmotic (200 mM mannitol) stress on the auxin metabolome by UHPLC-MS/MS, auxin distribution by confocal microscopy, and transcript levels of selected genes by qRT-PCR in Arabidopsis thaliana ecotype Columbia-0 (Col-0) and DR5rev::GFP (DR5) line. During long-term stress (13 days), a stability of the auxin metabolome and a tendency to increase indole-3-acetic acid (IAA) were observed, especially during salt stress. Short-term stress $(3 \mathrm{~h}$ ) caused significant changes in the auxin metabolome, especially $\mathrm{NaCl}$ treatment resulted in a significant reduction of IAA. The data derived from auxin profiling were consistent with gene expressions showing the most striking changes in the transcripts of YUC, GH3, and UGT transcripts, suggesting disruption of auxin biosynthesis, but especially in the processes of amide and ester conjugation. These data were consistent with the auxin distribution observed in the DR5 line. Moreover, $\mathrm{NaCl}$ treatment caused a redistribution of auxin signals from the quiescent center and the inner layers of the root cap to the epidermal and cortical cells of the root elongation zone. The distribution of PIN proteins was also disrupted by salt stress; in particular, PIN2 was suppressed, even after 5 min of treatment. Based on our results, the DR5 line was more sensitive to the applied stresses than Col-0, although both lines showed similar trends in root morphology, as well as transcriptome and metabolome parameters under stress conditions.
\end{abstract}

Keywords: abiotic stress; Arabidopsis thaliana; auxin distribution; auxin metabolome; auxin transcriptome; root growth

\section{Introduction}

As sessile organisms, plants have amazing adaptability to the ever-changing environment and are able to survive under often unpleasant conditions such as extreme temperatures, lack of water, increased soil salinity, etc. Understanding the mechanisms of adaptation to abiotic stress has been of great importance in the last decades in the context of the current climate changes and the predicted further warming and decrease in precipitation, especially in the arid and semi-arid Mediterranean region [1].

The plant root is very sensitive to the environment and is the first plant organ to be confronted with unpleasant environmental changes in the soil such as water deficit and increased salinity. Root responses to abiotic stress are very dynamic and complex and involve high plasticity in root growth and architecture. Root system architecture (RSA) is determined by primary root growth (PR), initiation and emergence rates for lateral root 
(LR) primordia, and growth of LR. Root growth and RSA are processes that are strongly regulated by the phytohormone auxin. The high auxin concentration in root quiescent cells and in the stem cell niche is essential for the coordination and establishment of the different root tissues and root growth [2,3]. Maintenance of the auxin maximum in the root tip is achieved by transport of shoot-derived auxin into the root tip, where complex patterns of auxin efflux carriers lead to auxin accumulation in the stem cell niche [4]. Moreover, local auxin biosynthesis is possible in all parts of the root system [5]. The amount of auxin in a particular cell type is crucial for cell fate. Thus, the accumulation of auxin in pericycle cells transforms them into lateral root founder cells, which further divide and form a lateral root primordium [6].

The major processes controlling root growth and development, such as cell division, elongation, and differentiation, are mediated by the formation of local auxin maxima and local auxin minima in root tissues. Dynamic auxin gradients essential for the integration of various environmental and endogenous signals are regulated by de novo auxin biosynthesis, hydrolysis of auxin conjugates, polar transport, auxin inactivation through various conjugation and oxidation pathways. Auxin metabolism and homeostasis have been extensively studied and numerous genes have been identified to date. A review of auxin homeostasis has been updated [7-12] and is summarized in Figure 1.

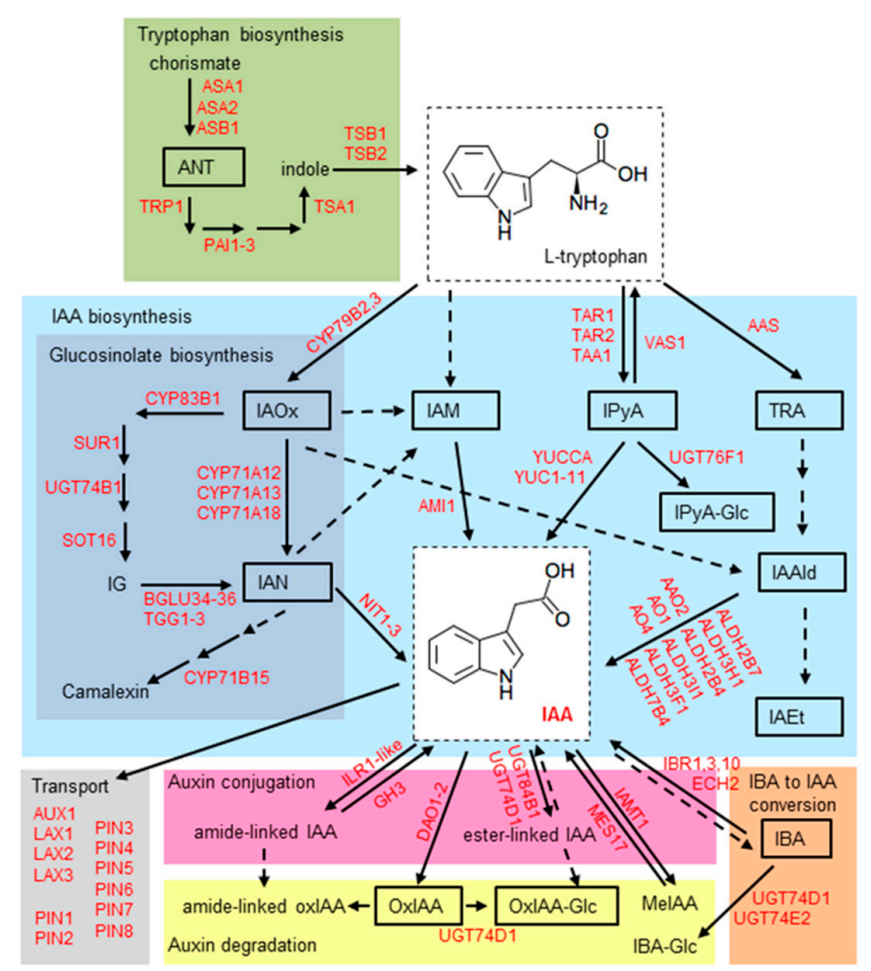

Figure 1. Scheme of IAA metabolism in Arabidopsis thaliana (Arabidopsis). The pathways involved in auxin metabolism in Arabidopsis are based on the KEGG pathways [13] and data published in [7,14-19]. The darker blue panel shows pathways specific for Arabidopsis, Brassica, and Sinapis [20]. Yellow and pink panels indicate pathways for IAA degradation [14] and IAA conjugation [15], respectively. The contribution of the IBA to IAA conversion to the active IAA pool is illustrated by the orange panel [16]. The green panel shows the synthesis of tryptophan from the precursor chorismate via the shikimate pathway [21]. Dashed arrows indicate pathways that may contain multiple enzymatic steps, and where the genes/enzymes are not known. ANT, anthranilate; IAA, indole-3-acetic acid; IAAld, indole-3-acetaldehyde; IAEt, indole-3-acetethanol; IAM, indole3-acetamide; IAN, indole-3-acetonitrile; IAOx, indole-3- acetaldoxime; IBA, indole-3-butyric acid; IBA-Glc, indole-3-butyryl-1-O- $\beta$-d-glucose; IPyA, indole-3-pyruvic acid; MeIAA, indole-3-acetic acid methyl ester; oxIAA, 2-oxoindole-3-acetic acid; oxIAA-Glc, 2-oxoindole-3-acetyl-1-O- $\beta$-d-glucose; TRA, tryptamine. 
Polar auxin transport enables the adequate distribution of auxin from the site of synthesis to the site of action, thus contributing to auxin homeostasis. Components that control auxin transport are the auxin influx carriers localized in the plasma membrane, the auxin permeases of the AUX1/LAX family, and efflux facilitators and transporters, the PIN family and $\mathrm{ABCB}$ family, respectively [22,23].

In addition to the role of auxin in regulating root growth and development under optimal conditions, which has been well studied previously (see review $[5,6]$ ), recent data show that auxin plays an important role in mediating changes in the root during abiotic stresses (see reviews $[4,24,25]$ ). Here, we focus on salinity and osmotic stress and their effects on root growth and RSA. Salinity stress is a complex stress consisting of ionic stress caused by toxic ion concentrations (mainly $\mathrm{Na}^{+}$), osmotic stress caused by the associated decreased water uptake, and oxidative stress caused mainly by the increase in reactive oxygen species levels (ROS) [26]. There is a partial overlap with drought responses due to the osmotic component. However, the changes in RSA show clear differences in PR growth, which is strongly promoted during drought, whereas it is inhibited during salt stress [4].

Studies in a variety of plant species have shown that auxin homeostasis is a direct link between growth regulation and stress adaptation responses (see review [27]). According to microarray data tracking the expression of YUCCA flavin monooxygenases (YUCs) (involved in the major IAA biosynthetic pathway in Arabidopsis), auxin biosynthesis is shifted from columella cells to the root epidermis during salt stress [4]. This redistribution of auxin maxima correlates with reduced growth of PR and LR and a decrease in the number of LRs [28-30]. In Brassica rapa seedlings, IAA precursor profiling showed that salinity stress activates the indole-3-acetamide (IAM) and indole-3-acetaldoxime (IAOx) biosynthetic pathways, while the indole-3-pyruvic acid (IPyA) pathway is repressed [31] It was shown that changes in CYP79B2 expression under salt stress positively correlated with the development of lateral roots in Arabidopsis, and cyp79b2 cyp79b3 double mutants developed fewer and shorter lateral roots under salt stress compared to control conditions, also suggesting that the IAOx biosynthetic pathway is more dominant under salt stress in Brassicaceae [32].

In addition to auxin biosynthesis, increased salinity and osmotic stress affect polar auxin transport. It has been shown that the root bends away from the salt under a saline environment (halotropism), which is caused by redistribution of auxin supported by changes in auxin transporters (AUX1, PIN1, PIN2) [24,33]. In addition, salt exposure reduces the size of the root meristem and inhibits the formation of LR due to decreased levels of PIN1, PIN3 and PIN7 proteins [34]. Auxin transport is also inhibited during drought stress by inhibiting PIN1 expression, which could facilitate increased downward bending of roots [34].

Looking at IAA conjugation, all GH3 enzymes involved and some UGTs seem to be highly expressed in roots and are upregulated during salt stress [4]. The oxidation process of IAA is very active in root tissues; the enzyme DAO1 catalyzes the temporal and tissue-specific oxidative inactivation of IAA, and the DAO1 gene is expressed in the cortical, endodermal, and pericycle cells of the distal elongation zone and maturation zone of the root, root columella, and atrichoblasts, while DAO2 is weakly expressed in the root columella and procambium of the root maturation zone [35]. The same authors showed that the $D A O 2$ knockdown exhibited increased lateral root density, indicating a possible role of IAA oxidation in the root response to salt stress [35].

Root growth is remodeled under salt and osmotic stress and the mechanisms of this remodeling are still unclear. In this work, we investigated the auxin metabolome in Arabidopsis (Col-0 and DR5 line) under short- and long-term abiotic stress conditions with $\mathrm{NaCl}$ and mannitol as salt and osmotic stressors, respectively. In addition, auxin distribution in root tissue of the DR5 line was examined after short-term (1.5-12 h) stressor treatments (salt and mannitol). As salt induced a more drastic redistribution of auxin, we further examined the stress-induced distribution of PIN proteins (PIN2, PIN4, and PIN7) using the Arabidopsis transgenic lines pPIN2::PIN2-GFP, $p$ PIN4::PIN4-GFP, and 
pPIN7::PIN7-GFP at 5 to 15 min after salt treatments. Finally, the expression of auxin related genes was analyzed and root growth, auxin metabolome data, and auxin distribution patterns were discussed in correlation with the transcript level of selected genes under saline and osmotic conditions.

\section{Results and Discussion}

\subsection{Root Growth under Abiotic Stress}

To investigate the effect of salt and osmotic stress on root growth, Arabidopsis Col-0 and the DR5 lines were used. Seedlings were germinated on MS medium for 4 days and then transferred to the same medium supplemented with $100 \mathrm{mM} \mathrm{NaCl}$ or $200 \mathrm{mM}$ mannitol for the next 13 days, with root growth monitored periodically. Both lines showed similar responses to the applied stressors (Figure 2, Figure S1).
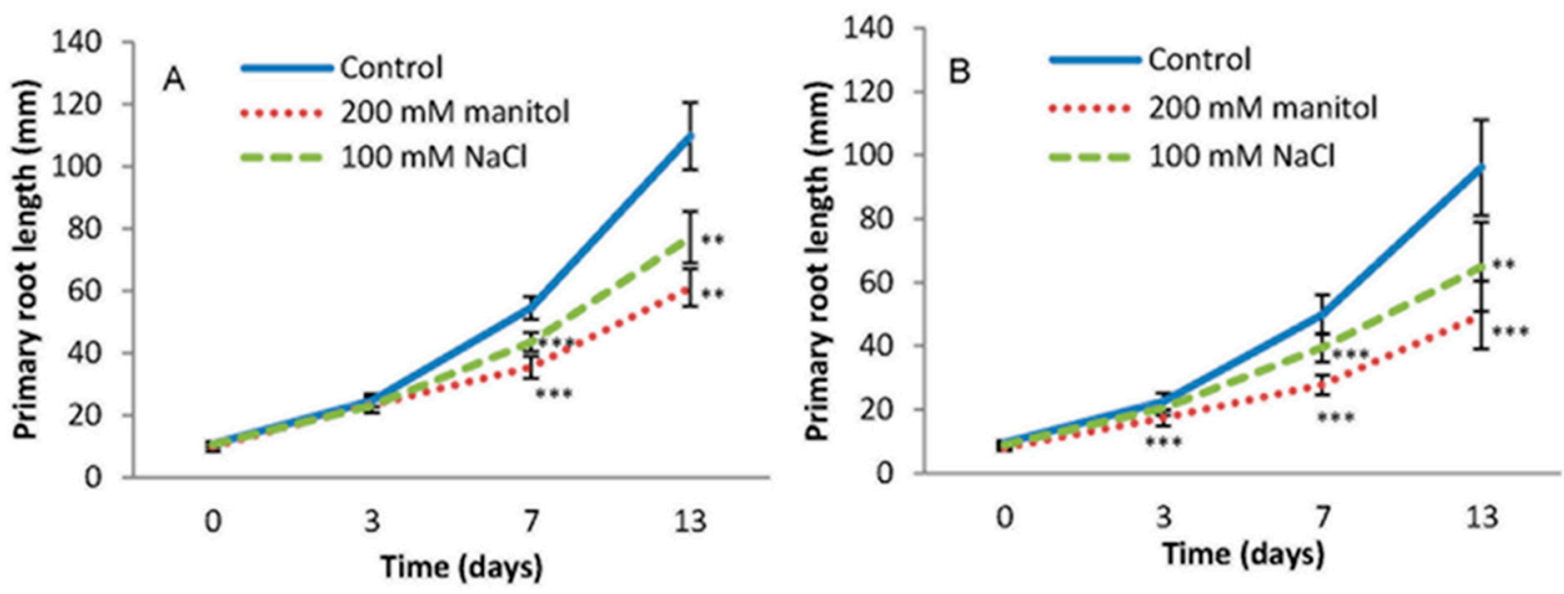

Figure 2. Inhibition of the primary root length of Arabidopsis thaliana Col-0 (A) and DR5 (DR5rev::GFP) line (B) grown on MS medium (Control) or MS medium supplemented with $100 \mathrm{mM} \mathrm{NaCl}(\mathrm{NaCl})$ and $200 \mathrm{mM}$ mannitol (Man). Primary root length was monitored for 13 days. The experiment was performed on three biological replicates, each consisted of 5 roots. Results are average \pm SD, $n=15$ seedlings. Asterisks indicate significant difference in primary root length in plants grown under stress conditions versus the corresponding control in a Student's $t$-test ${ }^{* *}$ and ${ }^{* * *}$ correspond to $p$-values of $0.01>p>0.001$ and $p<0.001$, respectively).

There were no visible differences in root growth and morphology for the first three days of treatments in Col-0, while DR5 showed a statistically significant reduction in root length after the treatments compared to the corresponding controls. The inhibition of primary root growth was more pronounced with the duration of treatments. The primary root length was inhibited by $44.4 \%$ and $29.6 \%$ in Col- 0 and $48.3 \%$ and $32.5 \%$ in the DR5 line after 13 days of treatments with mannitol and $\mathrm{NaCl}$, respectively, compared to the controls (Figure 2).

The occurrence of lateral roots (LRs) was significantly reduced in $\mathrm{NaCl}$ - and mannitolexposed seedlings during the studied period (Figure 3, Supplementary Figure 1). The average number of lateral roots was similar in both Arabidopsis lines under control conditions. Under salinity stress, the number of LRs in Col-0 and DR5 was significantly lower than in the corresponding controls. The effect of osmotic stress was even more drastic in both lines. The average total length of LRs also decreased under stress conditions in both lines, with osmotic stress having a greater effect (Figure 3). Thus, results indicate that stress conditions caused a similar inhibitory effect to LR numbers and total LR length in both lines. 

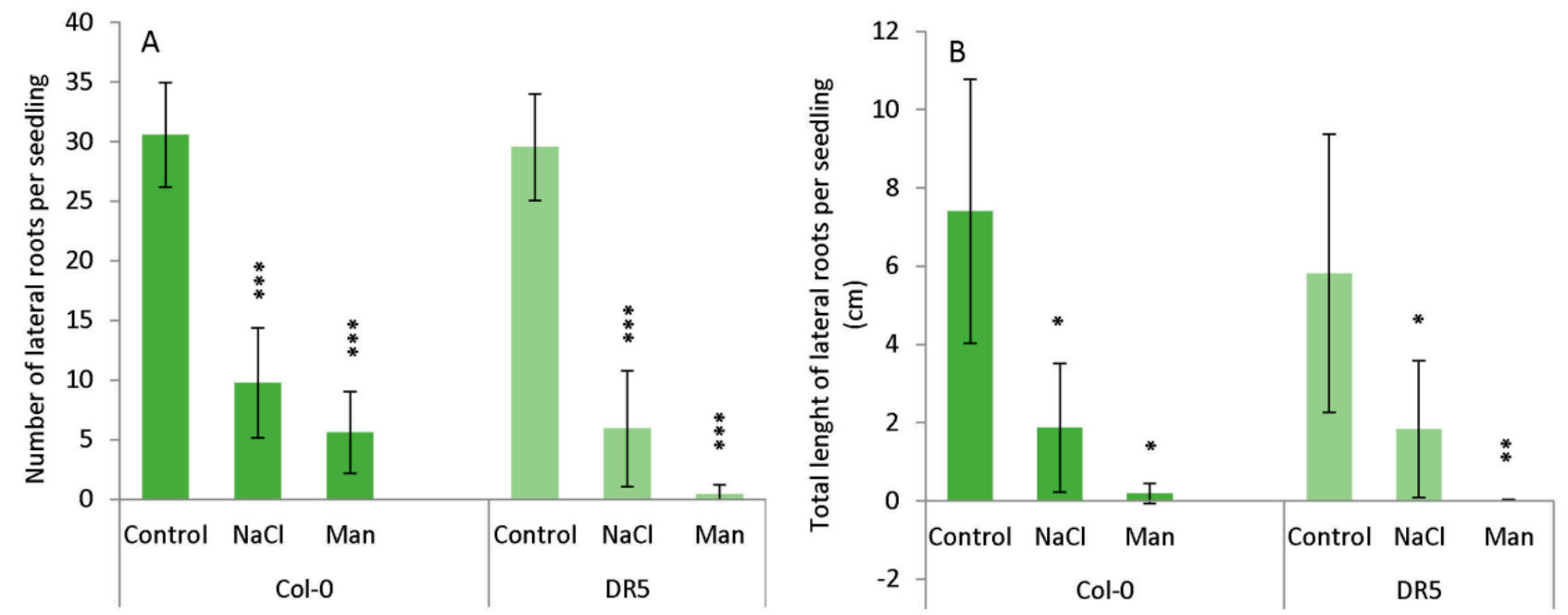

Figure 3. Lateral root (LR) number (A) and total length of LRs (B) in wild type Arabidopsis thaliana Col-0 and DR5rev::GFP line (DR5) upon 13-day growth on MS medium (Control) or MS medium supplemented with $100 \mathrm{mM} \mathrm{NaCl}(\mathrm{NaCl})$ or $200 \mathrm{mM}$ mannitol (Man). The experiment was performed on five biological replicates, each consisted of 5 roots. Results are expressed as mean $\pm \mathrm{SD}, n=25$ seedlings. Asterisks indicate significant difference in the measured parameters in controls compared to stresses conditions in each line in a Student's $t$-test $\left(^{*},{ }^{* *}\right.$, and ${ }^{* *}$ correspond to $p$-values of $0.1>p>0.01$, $0.01>p>0.001$, and $p<0.001$, respectively).

\subsection{Auxin Profile in Arabidopsis after Short-Term and Prolonged Stress Conditions}

It is well known that the plant hormone auxin is crucial for all aspects of plant development, both under optimal and stress conditions [36], especially for root growth and RSA [4-6]. Therefore, we performed auxin metabolomic analyses by liquid chromatographytandem mass spectrometry (LC-MS/MS) in both Col-0 WT and DR5 lines in 4-day-old seedlings after $3 \mathrm{~h}$ (Figure 4 ) and in Arabidopsis roots after 13 days of stress application (Figure 5). As can be seen in Figures 4 and 5, the changes in auxin metabolites in both Arabidopsis lines followed the same trend, although the concentrations of certain metabolites differed.

After short-term stress application (Figure 4), IAA content did not change under osmotic stress but decreased under salinity stress compared with the corresponding control, especially in the DR5 line (1.8-fold). Similarly, the content of ANT, the precursor of tryptophan (TRP), was significantly decreased under salinity treatment (2.4-fold in both lines), whereas TRP was significantly increased (2.6- and 2.3-fold in Col-0 and DR5 lines, respectively). The major IAA precursor, IPyA, was not significantly affected, although there is a tendency to decrease under salinity treatment in both lines. Interestingly, several other IAA biosynthetic pathways appear to be more strongly induced under salinity stress conditions. Similar to IAA, the levels of IAN were decreased under both stress conditions, but most significantly under salinity treatment (1.7- and 2.5-fold in Col-0 and DR5 lines, respectively). However, the IAM content was increased about 3-fold under salinity stress in the DR5 line compared with the control. Auxin metabolite content was also negatively affected after short-term stress application. The levels of both amino acid conjugates, IAA-aspartate (IAA-Asp) and IAA-glutamate (IAA-Glu) were significantly decreased upon salinity stress in both lines. Interestingly, the DR5 line contained approximately 2 -fold higher levels of IAA-Asp and IAA-Glu under control conditions compared with Col-0. The auxin catabolites oxIAA and its glucosyl ester (oxIAA-Glc) were each slightly decreased under salinity and osmotic stress. IAA glucosyl ester (IAA-Glc) also changed significantly in Col-0 under both stress treatments, whereas it remained unchanged in the DR5 line. 


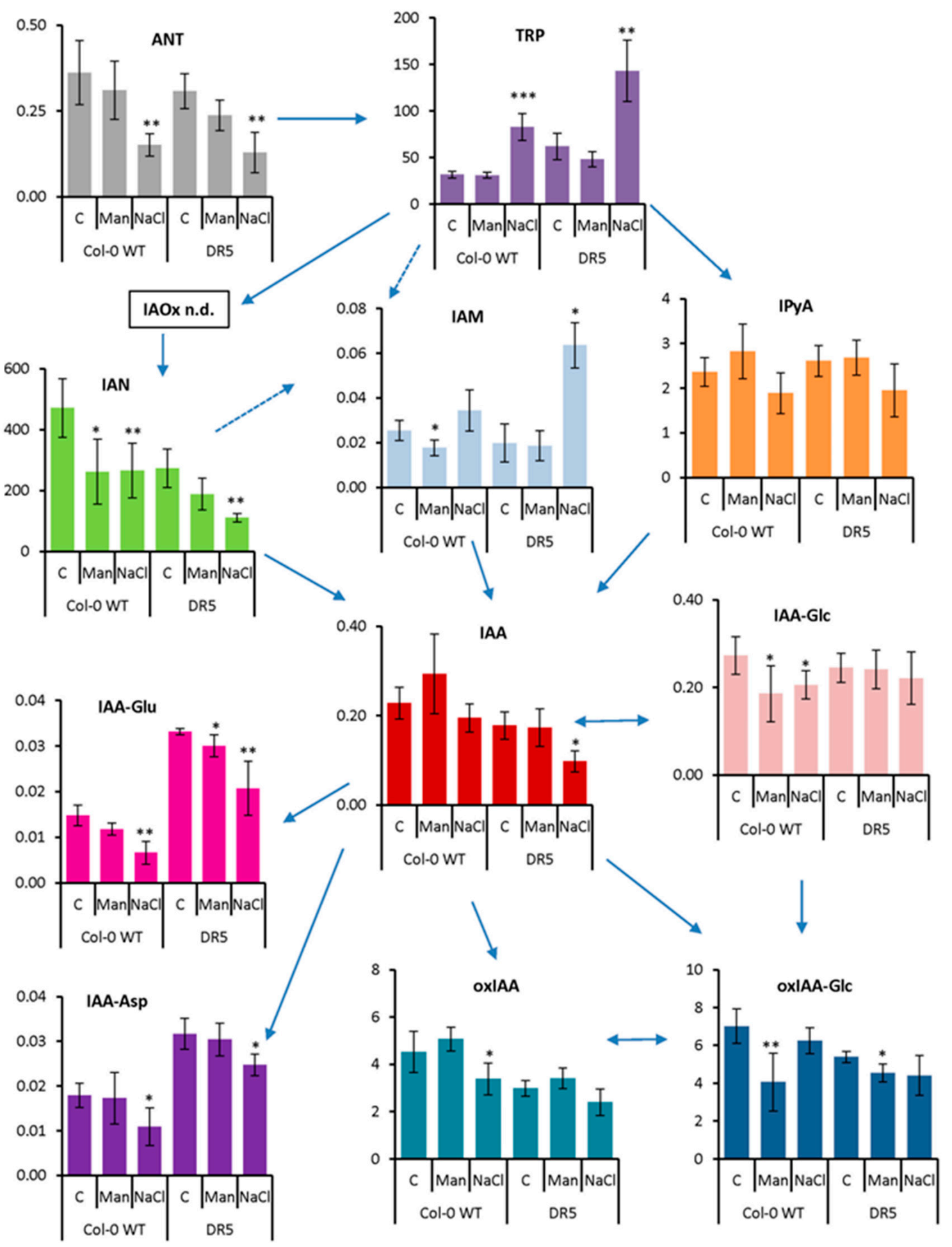

Figure 4. Auxin metabolome profile measured in 4-day-old seedlings of Arabidopsis thaliana Col-0 and DR5rev::GFP line (DR5) treated with $200 \mathrm{mM}$ mannitol (Man) or $100 \mathrm{mM} \mathrm{NaCl}(\mathrm{NaCl})$ for $3 \mathrm{~h}$ compared to the corresponding controls (C). Metabolite levels are expressed in $\mathrm{nmol} \mathrm{g}^{-1} \mathrm{FW}$. Results are average $\pm \mathrm{SD}, n=3$. Asterisks indicate significant difference in the levels under stress conditions versus the control in a Student's $t$-test $\left({ }^{*},{ }^{* *}\right.$, and ${ }^{* * *}$ correspond to $p$-values of $0.05>p>0.01$, $0.01>p>0.001$, and $p<0.001$, respectively). ANT, anthranilate; IAA, indole-3-acetic acid; IAA-Asp, indole-3-acetyl-Laspartic acid; IAA-Glc, indole-3-acetyl-1-O- $\beta$-d-glucose; IAA-Glu, indole-3-acetyl-L-glutamic acid; IAM, indole-3-acetamide; IAN, indole-3-acetonitrile; IAOx, indole-3-acetaldoxime; IPyA, indole-3-pyruvic acid; oxIAA, 2-oxoindole-3-acetic acid; oxIAA-Glc, 2-oxoindole-3-acetyl-1-O- $\beta$-d-glucose; TRP, tryptophan; n.d., not detected. 

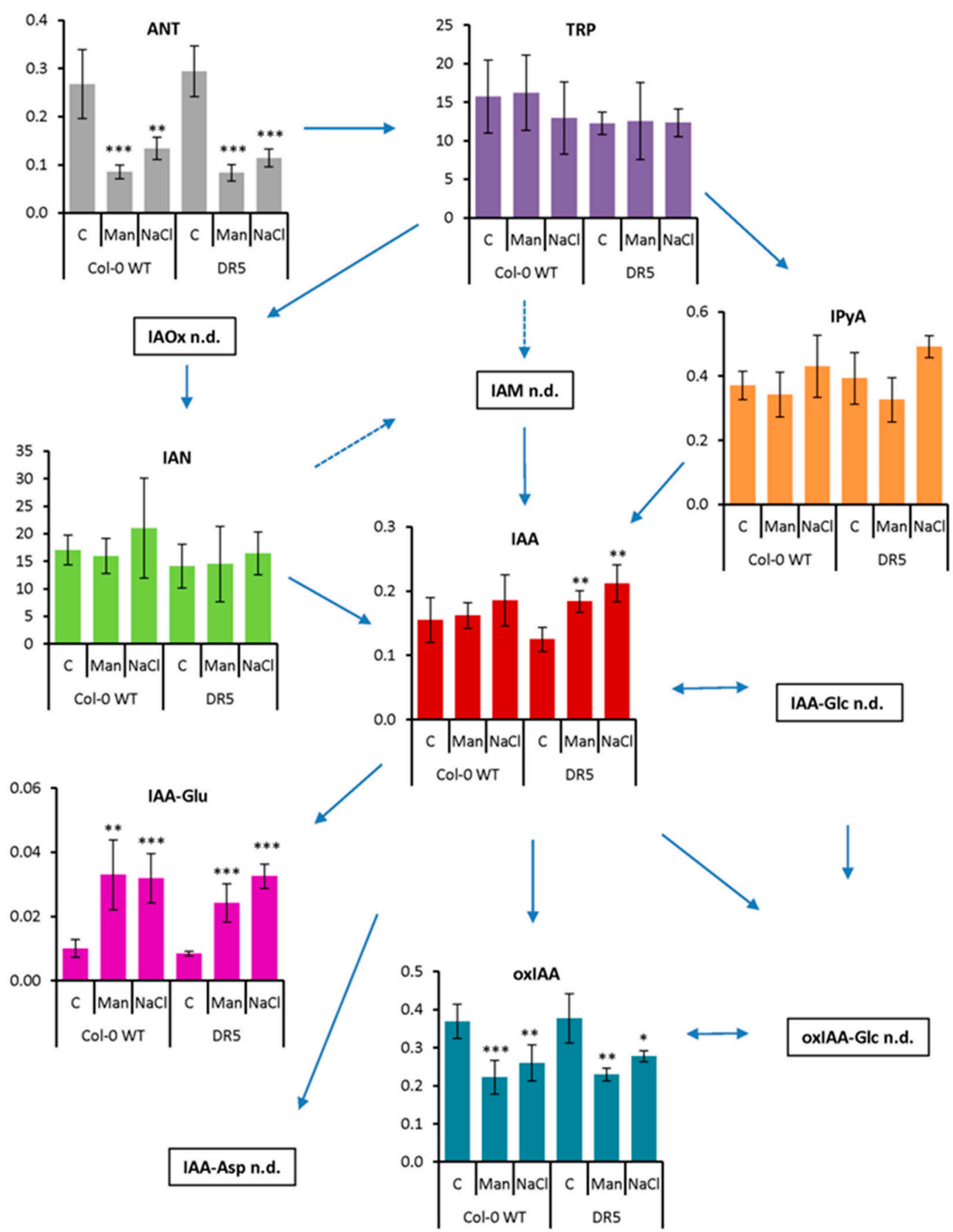

Figure 5. Auxin metabolome profile measured in the roots of Arabidopsis thaliana Col-0 and DR5rev::GFP line (DR5) after 13-day treatment with $200 \mathrm{mM}$ mannitol (Man) and $100 \mathrm{mM} \mathrm{NaCl}(\mathrm{NaCl})$ compared to the corresponding control (C). Metabolite levels are expressed in $\mathrm{nmol} \mathrm{g}^{-1} \mathrm{FW}$. Results are average $\pm \mathrm{SD}, n=3$. Asterisks indicate significant difference in the levels under stress conditions versus the control in a Student's $t$-test $\left({ }^{*}, * *\right.$, and ${ }^{* * *}$ correspond to $p$-values of $0.05>p>0.01,0.01>p>0.001$, and $p<0.001$, respectively). Abbreviations: ANT, anthranilate; IAA, indole-3-acetic acid; IAA-Asp, indole-3-acetyl-L-aspartic acid; IAA-Glc, indole-3-acetyl-1-O- $\beta$-d-glucose; IAA-Glu, indole-3-acetyl-L-glutamic acid; IAM, indole-3-acetamide; IAN, indole-3-acetonitrile; IAOx, indole-3-acetaldoxime; IPyA, indole-3-pyruvic acid; oxIAA, 2-oxoindole-3-acetic acid; oxIAA-Glc, 2-oxoindole-3-acetyl-1-O- $\beta$-d-glucose; TRP, tryptophan; n.d., not detected.

As RSA was severely affected by the prolonged osmotic and salinity stress (Figures 2 and 3), we also performed auxin metabolome analysis in the roots of the 13-day-treated Arabidopsis plants and compared them with the auxin metabolome under the control conditions (Figure 5). 
Our results show that the content of ANT in both lines was significantly decreased in both prolonged stress treatments, while the content of TRP did not change. However, two detected IAA precursors, IAN and IPyA, showed a tendency to increase in $\mathrm{NaCl}$ treatments, although the changes were not statistically significant. Similarly, IAA also showed a tendency to increase in both lines under both stress conditions, but statistically significant changes were obtained only in the DR5 line compared with the control. These results are consistent with an increase in IAA concentration in Chinese cabbage seedlings after $24 \mathrm{~h}$ of salinity stress [31]. Importantly, the process of amide conjugation was strongly induced under both stress conditions. The IAA-Glu levels were significantly increased, indicating active auxin homeostasis to regulate optimal IAA levels. On the other hand, the oxIAA levels were significantly decreased under osmotic and salinity stress conditions in both lines. Furthermore, glucosyl ester conjugates were not detected after long-term stress application. Similar patterns of IAA-Glu and oxIAA concentrations were previously published in root and shoot tissues of control and salt-stressed Arabidopsis seedlings [37].

\subsection{Early Auxin Redistribution in Root under Stress Conditions}

Since we obtained the changes in the auxin metabolome in Arabidopsis after only $3 \mathrm{~h}$ of stress application, we further followed auxin distribution in the roots of 4-dayold seedlings in the range of treatments from 1.5 to $12 \mathrm{~h}$ by using the artificial reporter line DR5rev::GFP (DR5), which is the most commonly used tool for monitoring auxin distribution in planta. The most striking changes were observed in the salinity treatment, while the mannitol treatment did not cause significant auxin redistribution in the root compared with the corresponding control. This observation was in agreement with LCMS/MS measurements under short-term stress, which also indicated less pronounced changes in the auxin metabolome under osmotic than under salinity stress (Figure 4). In control and mannitol-treated seedlings, auxin signals were concentrated in the quiescent center and in the internal layers of the root cap after $3 \mathrm{~h}$ (Figure 6, Video S1).

Exposure to $100 \mathrm{mM} \mathrm{NaCl}$ for $3 \mathrm{~h}$ caused a significant reduction of auxin in the quiescent center and in the internal layers of the root cap (Figure 6), whereas an increase of auxin was detected in the epidermal and cortex cells of the root elongation zone (Figure 7, Video S3). A similar observation was reported by Wang et al. [28] using the DR5::uidA line under prolonged $(48 \mathrm{~h})$ salt treatment $(150 \mathrm{mM} \mathrm{NaCl})$; where GUS expression is mainly distributed in the epidermal cells of the root, while the level of GUS staining is significantly reduced in the area around the quiescent center. Interestingly, as shown here, the redistribution of auxin under salinity stress is much faster than previously reported [28] At the same time point, osmotic stress did not alter auxin accumulation or distribution compared to the corresponding control. Osmotic stress induced by mannitol increased auxin accumulation in the epidermis and provascular tissue, but auxin remained high in the quiescent center and columella (Figure 6, Video S2).

\subsection{PINs Redistribution under Salinity Stress}

As salt stress drastically affected the redistribution of auxin in different cell layers of the root elongation zone, we additionally investigated the occurrence of PINs in roots upon salt application. Previously, it was reported that reduced auxin accumulation in roots subjected to salt stress could be due to the suppression of auxin carriers in response to salt concentration and duration of stress application [34]. Most of the PIN proteins are located in the plasma membrane of root cells and are restricted to specific areas of the cell that mediate directional auxin fluxes within the tissue, influencing auxin distribution and thus root growth and architecture [38]. To investigate the effect of salt on the redistribution of PIN proteins, we used the transgenic Arabidopsis lines $p$ PIN2::PIN2-GFP, $p$ PIN4::PIN4-GFP, and $p$ PIN7::PIN7-GFP. 

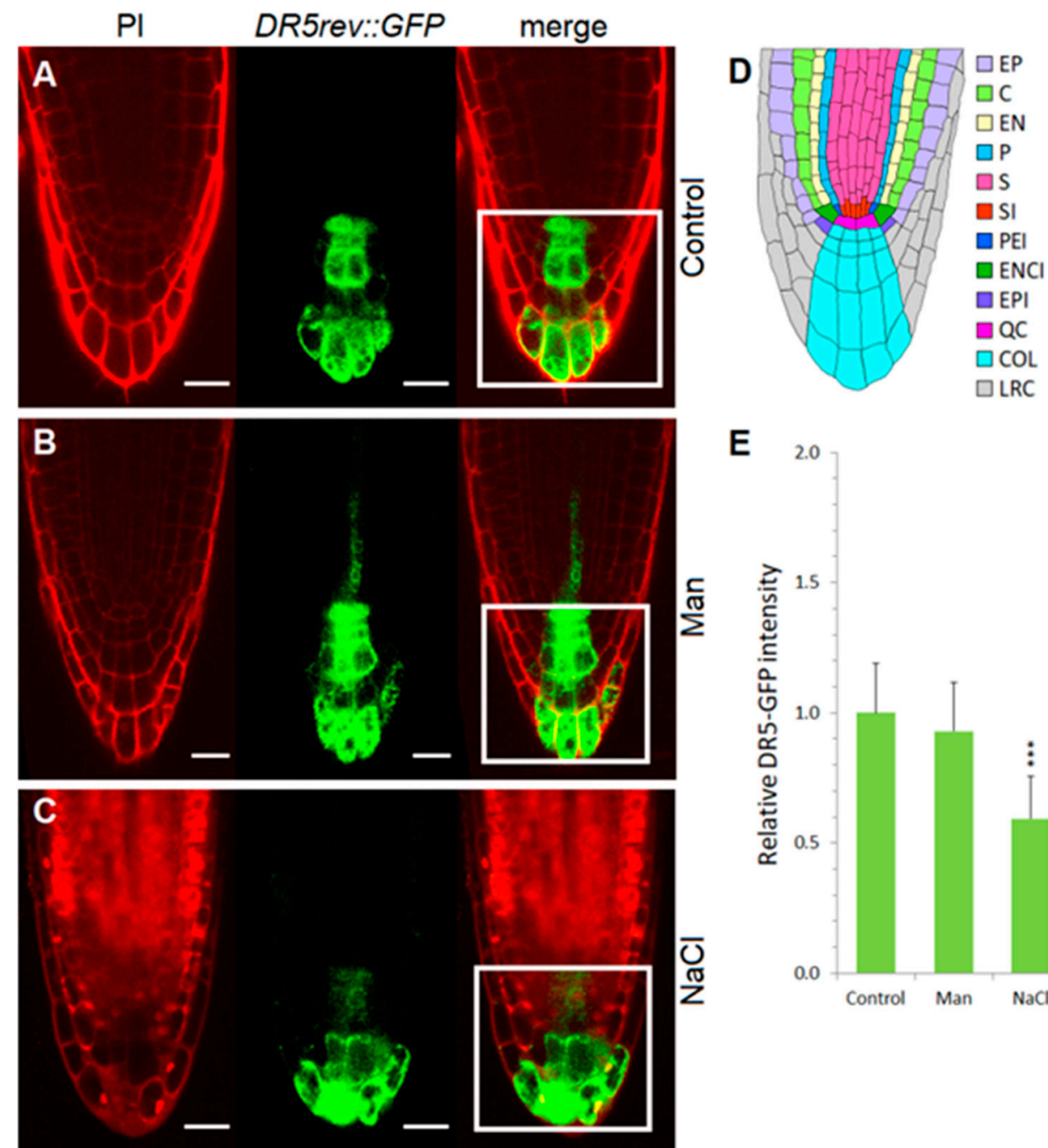

E

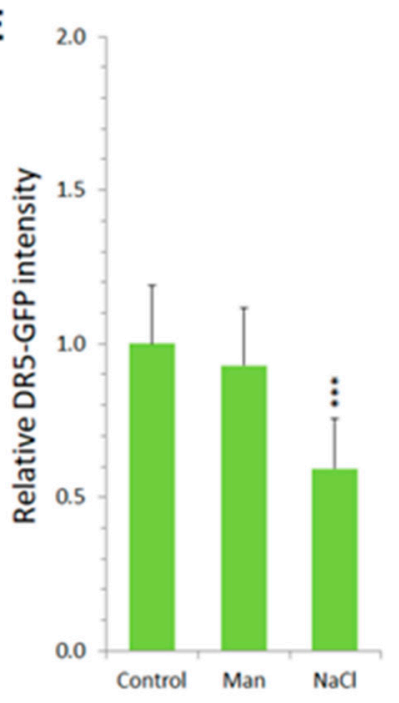

Figure 6. Auxin distribution and relative GFP intensity in the root tips of DR5rev::GFP line. Seedlings were exposed for $3 \mathrm{~h}$ to $2.5 \mathrm{mM}$ MES (control) (A) or control medium was supplemented with $200 \mathrm{mM}$ mannitol (Man) (B) or $100 \mathrm{mM} \mathrm{NaCl}(\mathrm{NaCl})$ (C). GFP fluorescence was monitored with the laser scanning confocal microscope and its intensity was quantified. The anatomy of the root tip is shown in (D). The area of interest was selected with the ImageJ rectangle ROI selection tool (white rectangle) in at least 10 roots. Fluorescence intensity (E) of these ROIs was measured as integrated density and the relative intensity, presented in the histogram, was calculated as the value of integrated density normalized to control without stressor; error bars represent SD. Asterisks $\left(^{* * *}\right)$ indicate significant differences with respect to the corresponding control (Student's $t$ test, $p<0.001$ ). Abbreviations: PI, propidium iodide; E, epidermis; C, cortex; EN, endodermis; P, pericycle; S, stele; SI, stele initials; PEI, pericycle initials; ENCI, endodermis/cortex initials; EPI, epidermis initials; QC, quiescent center; COL, columella; LRC, lateral root cap. Bar: $20 \mu \mathrm{m}$.

PIN2 is known to be localized to the underside of root cortical cells and to the upper surface of epidermal cells and to act primarily in the redistribution of auxin during root gravitropism. Immediately after $\mathrm{NaCl}$ application, PIN2 levels decreased and remained reduced during the next 15-min of treatment (Figure 8, Figure S2). This was consistent with the results obtained on the DR5 line after $\mathrm{NaCl}$ treatment, which showed auxin accumulation in the root cortex and epidermis (Figure 6). These results suggest a crucial role of relocalization of PIN2 protein in the overaccumulation of auxin in the root cortex and epidermis after initial salt stress. These results are consistent with the data of GalvanAmpudia and Testerink [33] who indicated that downregulation of PIN2 is induced when the intracellular $\mathrm{Na}^{+}$level in the root increases, resulting in decreased basipetal auxin 
transport in the root epidermis and lateral root cap. A similar observation of a more diffuse distribution of PIN2 is reported for 12 to $24 \mathrm{~h}$ of salt-treated Arabidopsis roots [39].

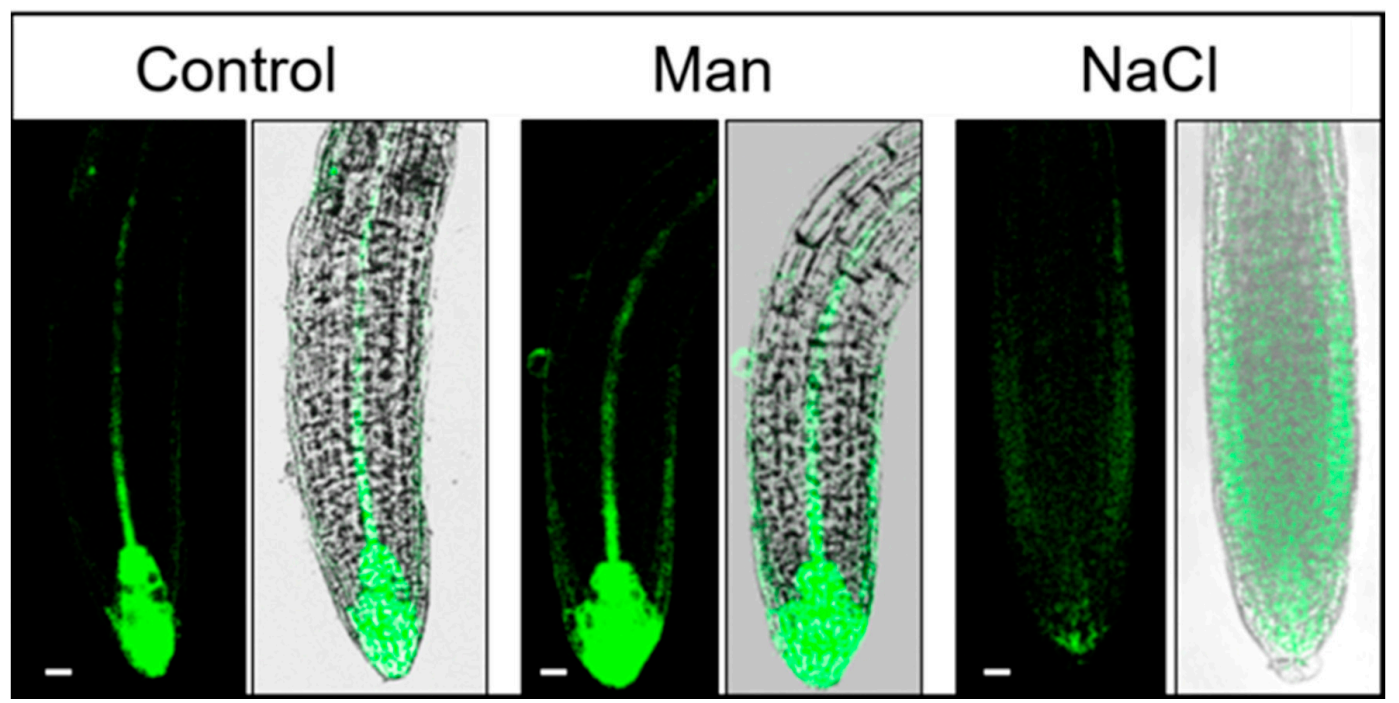

Figure 7. Auxin distribution in the Arabidopsis root of the DR5 line $6 \mathrm{~h}$ after treatment. Seedlings were exposed to $2.5 \mathrm{mM}$ MES (control) or control medium supplemented with $200 \mathrm{mM}$ mannitol or $100 \mathrm{mM} \mathrm{NaCl}$. GFP fluorescence was imaged with a Leica TCS SP8 X laser scanning confocal microscope. Bar: $10 \mu \mathrm{m}$.

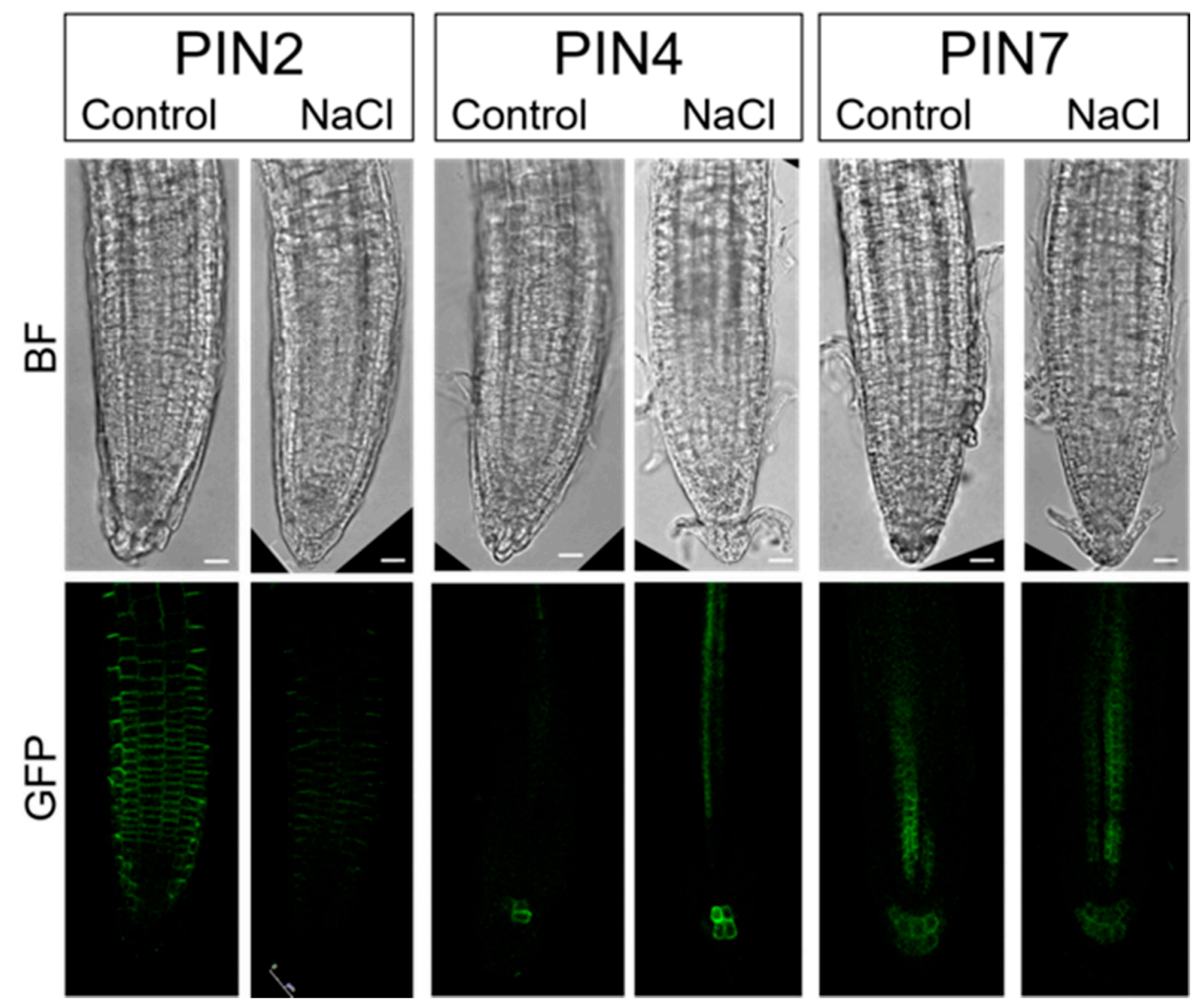

Figure 8. PIN proteins (PIN2, PIN4, and PIN7) distributed in the root tips of Arabidopsis $p$ PIN2::PIN2-GFP, $p$ PIN4::PIN4GFP, and $p P I N 7:: P I N 7-G F P$ lines before salt stress application (Control) and in response to immediate (10 min) salt stress $(100 \mathrm{mM} \mathrm{NaCl})$. GFP fluorescence was imaged with a laser scanning confocal microscope. Bar: $10 \mu \mathrm{m}$. 
As we found reduced auxin levels in and around the quiescent center of the DR5 line after salt stress, we also examined PIN4 and PIN7, which were previously reported to direct auxin toward the quiescent center. PIN4 is reported to localize to the basal side of cells in the central root meristem and with less pronounced polarity in the cells of the quiescent center while PIN7 is localized to the basal side of the stele cells and apolar in columella cells [40]. However, there was no change in the localization and intensity of PIN4 and PIN7 around the quiescent center immediately after salt treatment (Figures S3 and S4). Thus, the reduced auxin level in the quiescent center after short-term stress application is probably not associated with PIN4 and PIN7. However, immediately after salt stress application, we detected a stronger signal from PIN4 in stele cells (Figure 8, Figure S3).

Published data showed that prolonged salt stress $(6 \mathrm{~h})$ significantly reduced the transcript levels and fluorescence intensities of PIN1, PIN3, and PIN7 in roots, although their distribution patterns were not altered after $6 \mathrm{~h}$ of salt stress [34]. Previously, $\mathrm{NaCl}$ treatment was shown to strongly suppress the expression levels of PIN1, PIN2, PIN3, and DR5 after 5 days of treatment [41]. A decrease in auxin concentration in quiescent center cells may occur in other ways, by conjugation or degradation of auxin, or by efflux through other transporters. According to recent publications, certain genes involved in auxin homeostasis, such as IAR4, are induced to prevent $\mathrm{NaCl}$ overaccumulation [41]. The authors showed that $\mathrm{NaCl}$ accumulation promotes the production of ROS, which negatively modulates root growth by regulating PIN-mediated polar auxin transport in roots.

\subsection{Effect of Stress Conditions on the Expression of Genes Involved in Auxin Metabolism}

IAA levels are tightly regulated by numerous processes such as IAA biosynthesis, esterand amide-conjugation, conjugate hydrolysis, degradation, and auxin transport, as shown in Figure 1. For a deeper understanding of the observed changes in the auxin metabolome and auxin distribution under salinity and osmotic stresses, we analyzed the transcript levels of selected genes involved in auxin metabolism (tryptophan aminotransferase related 2 (TAR2, At4g24670); flavin-binding monooxygenase family protein (YUC5, At5g43890); auxin amidohydrolase (IAR3, At1g51760); auxin amidosynthetases (GH3.1, At2g14960; GH3.3, At2g23170; (GH3.12, At5g13320); uridine diphosphate glycosyltransferase 74E2 (UGT74E2, At1g05680); 2-oxoglutarate and Fe(II)-dependent oxygenase 2 (DAO2, At1g14120), and the auxin transport proteins PIN2 (At5g57090) and PIN4 (At2g01420) in the wild-type of the Col-0 ecotype and the DR5 line (Figure 9). The chromosomal locations for the selected genes studied are shown in Supplementary Materials Figure S5.

Targets for gene expression analysis were selected in accordance with the observed changes in our auxin profile and based on the transcriptome dataset [42] available on the eFP browser website [43]. Transcriptome dataset of the Arabidopsis Col-0 roots (stage 1.02 , [44]) treated with $150 \mathrm{mM} \mathrm{NaCl}$ and $300 \mathrm{mM}$ mannitol for the period of $0.5-24 \mathrm{~h} \mathrm{[42]}$ was used to generate a heat map of auxin-related gene expressions (Supplementary Materials Table S1). The expression patterns of the above dataset showed that salinity stress induced more pronounced changes in auxin-related genes than osmotic stress. This is in general agreement with our auxin metabolome data (Figure 4). Certain differences in the expression of selected genes were observed among the Arabidopsis lines studied (Figure 9).

The transcript level of the gene YUC5, whose protein product is involved in the IPyA auxin biosynthetic pathway, was increased approximately 1.8- and 1.6-fold under osmotic and salinity stress, respectively, in the Col-0. However, in the DR5 line, expression was down-regulated under osmotic stress and slightly up-regulated under salinity stress compared with untreated controls. This observation is consistent with the auxin level measured in 4-day-old Arabidopsis seedlings in these two lines (Figure 4), which is generally lower in the DR5 line compared with Col-0. Based on the transcriptomic data, it appears that YUC5 has a major role in Arabidopsis root auxin biosynthesis immediately after stressor application. 

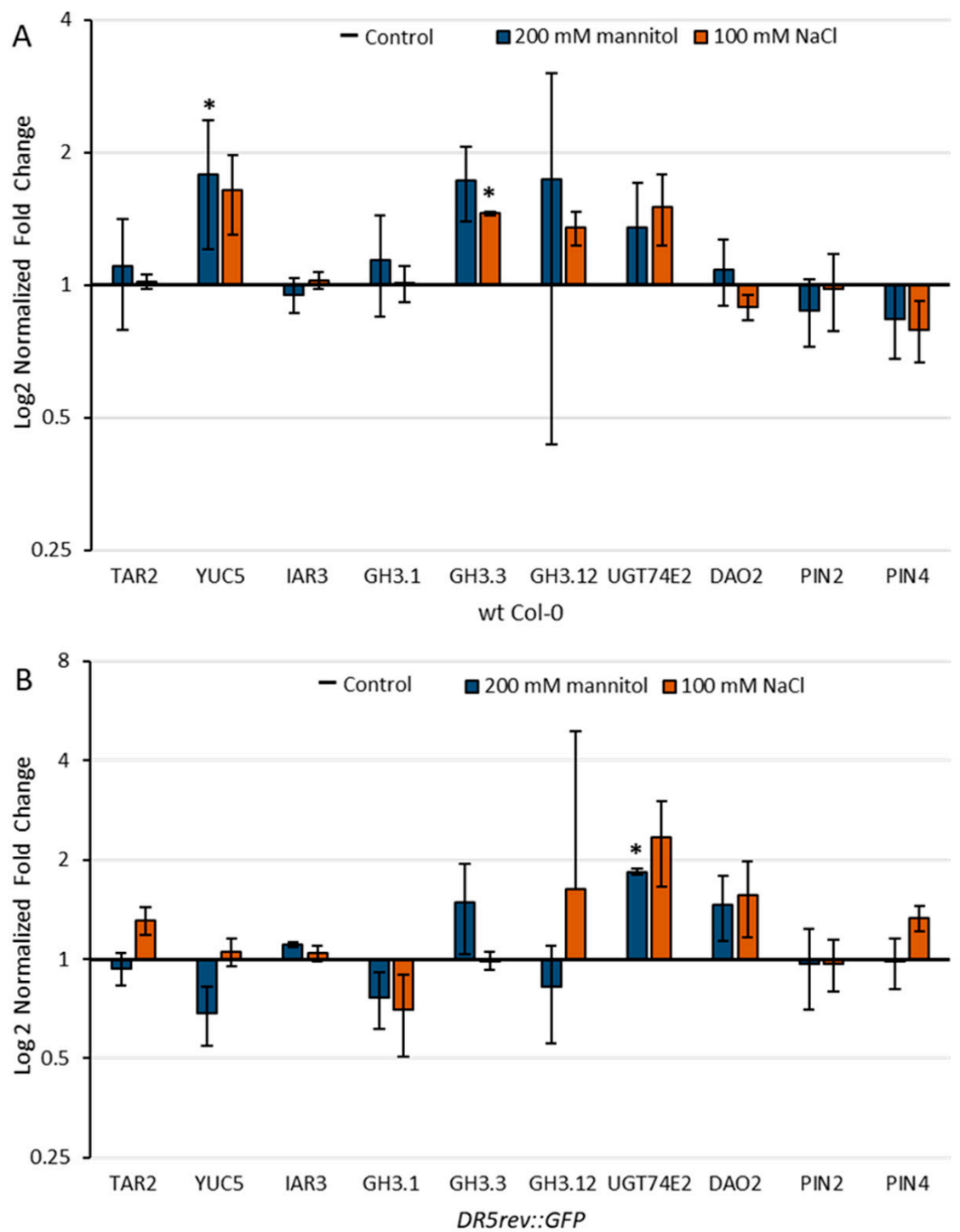

Figure 9. Relative gene expression of tryptophan aminotransferase related 2 (TAR2, At4g24670); flavin-binding monooxygenase family protein (YUC5, At5g43890); auxin amidohydrolase (IAR3, At1g51760); auxin amidosynthetase (GH3.1, At2g14960); auxin amidosynthetase (GH3.3, At2g23170); auxin amidosynthetase (GH3.12, At5g13320); uridine diphosphate glycosyltransferase 74E2 (UGT74E2, At1g05680); 2-oxoglutarate and Fe(II)-dependent oxygenase 2 (DAO2, At1g14120), and auxin transport proteins PIN2 (At5g57090) and PIN4 (At2g01420) in wild-type Arabidopsis Col-0 (A) and DR5 line (B) upon $3 \mathrm{~h}$ exposure to $100 \mathrm{mM} \mathrm{NaCl}$ or $200 \mathrm{mM}$ mannitol compared to the control measured by qPCR. Gene expression was calculated according to Livak [45] and presented as fold change compared to the controls. Asterisk $\left(^{*}\right)$ indicates significant differences with respect to the corresponding control (Student's $t$ test, $p<0.05$ ). Bars show means \pm SE of three biological replicates.

A similar effect with YUC genes has also been described in cucumber under salt stress, where CsYUC10b is strongly up-regulated and CsYUC10a and CsYUC11 are strongly down-regulated. Moreover, overexpression of CsYUC11 results in higher auxin levels in transgenic Arabidopsis and increases plant tolerance to salt stress as measured by root elongation [46]. Increased auxin levels in the plant have also been shown to increase the ability to cope with drought stress. Arabidopsis seedlings constitutively overexpressing $i a a M$ and thus having higher endogenous auxin content showed higher resistance to drought stress compared to wild-type plants. Moreover, the yuc1 yuc2 yuc6 triple mutant is more sensitive to drought than wild-type plants [47]. Similarly, transgenic poplar and potato plants overexpressing Arabidopsis YUC6 showed increased resistance to abiotic 
stress $[48,49]$. The increased expression of the YUC gene, as well as the higher IAA level in Col-0 compared to DR5, correspond to more efficient lateral root induction of the Col-0 line than of the DR5 line under stress conditions (Figures 2 and 3).

According to transcriptomic analysis, the most impaired process of auxin homeostasis under stress conditions was the synthesis of amide and ester conjugates (Supplementary Materials Table S1). It has been previously shown that GH3-mediated auxin homeostasis is an important part of the complex network of auxin actions that regulate stress adaptation in Arabidopsis [50]. Recently published work based on transcriptomic data and qRTPCR results indicate that GH3s play important roles under salinity and osmotic stress in different plant species [51-53]. Importantly, the significant increase in IAA-Glu levels observed in Arabidopsis wild type roots stressed for 13-days (Figure 5) is consistent with the transcriptome data (Figure 9). Under our experimental conditions, GH3.1, GH3.3, and GH3.12 were up-regulated by both stressors in Col-0, whereas DR5 surprisingly showed some differences in the expression of GH3s. In the stress-treated DR5 line, GH3.3 was mainly up-regulated under osmotic stress, GH3.12 under salinity stress, and GH3.1 was down-regulated under both stressors compared with the untreated control seedlings.

IAR3, the auxin amidohydrolase representative that can contribute to free IAA level by hydrolysis of amide conjugates, was slightly up-regulated in the stress treated DR5 line compared with the control. Kinoshita et al. [54] suggested that activation of IAR3 contributes to free auxin mediating root architecture under water limitation. It was also shown that the level of IAR3 transcript was increased in Arabidopsis due to high osmotic stress.

In addition, the UGT74E2 gene involved in ester conjugation of IAA was overexpressed in both lines under stress conditions, but more clearly in the DR5 line. In a recent publication, UGT74E2 and UGT74D1, among others, were identified as UGT candidates that may increase tolerance to salinity and drought stress in Brassica and Arabidopsis [55]. However, we could not detect increased IAA-Glc level under stress conditions (Figures 4 and 5) probably due to back-cleavage to the IAA.

$\mathrm{DAO} 2$ is involved with DAO1 in the degradation and oxidation of IAA to oxIAA. The $D A O 2$ gene is slightly up-regulated in the DR5 line under stress conditions compared with the control, although we did not detect increased levels of oxIAA in the seedlings under stress conditions. On the other hand, a reduction in oxIAA levels was also detected in Arabidopsis roots after $48 \mathrm{~h}$ exposure to salinity stress [37].

Most genes for transport proteins were slightly down-regulated under stress conditions (Supplementary Materials Table S1) [34,41]. Under our experimental conditions, the PIN4 transcript level was increased in the DR5 line compared to the control, which is consistent with the stronger fluorescence signal observed under salt stress (Figure 8).

Figure 10 shows the comparison of selected gene expressions between Col- 0 and DR5.

It can be seen that the transcript levels of most of the genes examined were lower in the DR5 line compared to Col-0 (TAR2, YUC5, IAR3, GH3.1, UGT74E2, PIN2, and PIN4). Only $D A O 2$ showed higher expression in the DR5 line under stress conditions. Interestingly, we observed that GH3.3 and GH3.12 showed higher transcript levels in the DR5 line compared with the Col-0 under control conditions, which is in agreement with higher levels of amide conjugates (IAA-Asp and IAA-Glu) in DR5 control seedlings compared with Col-0 control seedlings. In conclusion, higher transcript levels of GH3s and DAO2 genes in the DR5 line indicate that this line is more sensitive than Col-0 under stress conditions, consistent with the root growth assay (Figures 2 and 3).

The qPCR data are consistent with the auxin profile and root growth assay results in both lines (Figures 2, 3 and 5). Although the DR5 line appeared to be more sensitive compared with the wild-type Col-0 under our experimental conditions, similar trends of the morphological, metabolome and transcriptome parameters were observed in both lines under both stress conditions. Both stressors caused significant inhibition in main root growth as well as LRs number and total LRs length in both lines compared to their corresponding controls. Moreover, a similar trend of IAA increase was observed in both lines under both stress conditions and amide conjugation was strongly induced under 
both stress conditions. By taking all these into consideration, we would conclude that the DR5 line shows some aberrations in lateral root production under salt and osmotic stress but it is a useful and reliable tool for visualizing auxin distribution under different experimental setups.

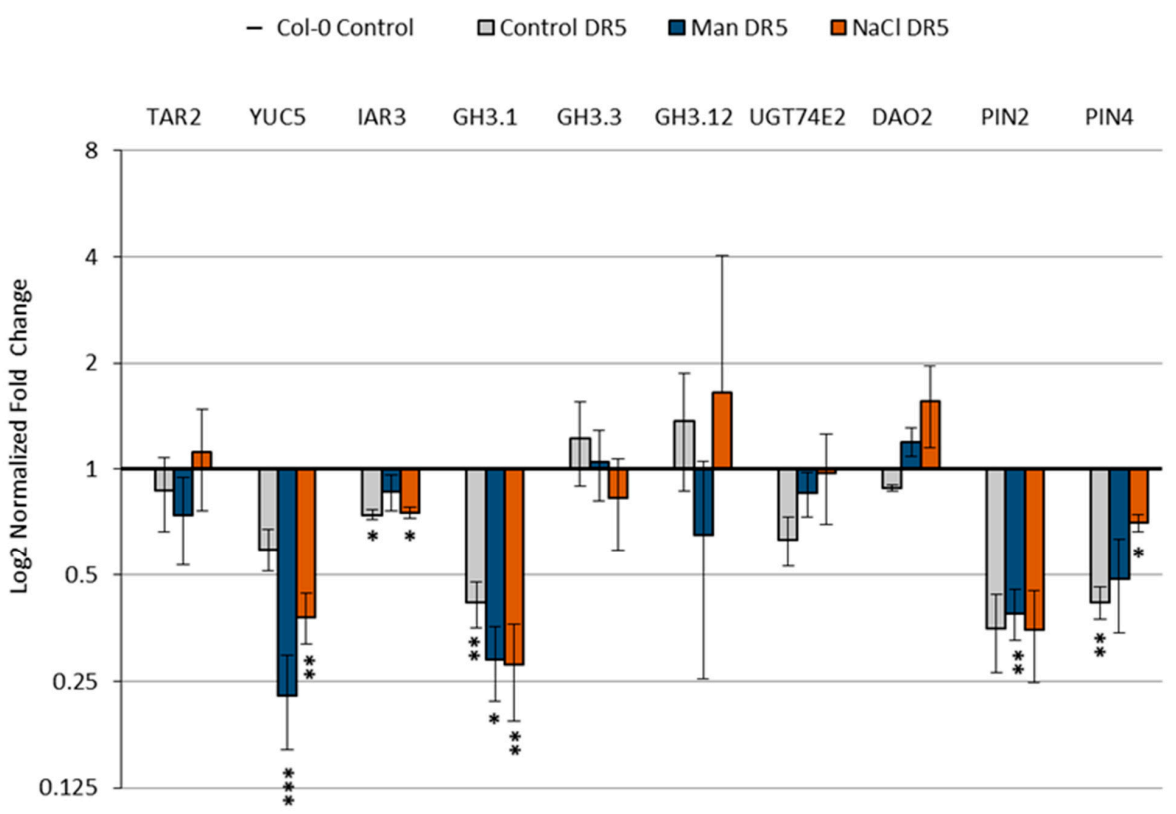

Figure 10. Relative gene expression in Arabidopsis DR5 (DR5rev::GFP) line upon $3 \mathrm{~h}$ exposure to $100 \mathrm{mM} \mathrm{NaCl}$ or $200 \mathrm{mM}$ mannitol compared to the Col-0 in each of the tested conditions of: tryptophan aminotransferase related 2 (TAR2, At4g24670); flavin-binding monooxygenase family protein (YUC5, At5g43890); auxin amidohydrolase (IAR3, At1g51760); auxin amidosynthetase (GH3.1, At2g14960); auxin amidosynthetase (GH3.3, At2g23170); auxin amidosynthetase (GH3.12, At5g13320); uridine diphosphate glycosyltransferase 74E2 (UGT74E2, At1g05680); 2-oxoglutarate and Fe(II)dependent oxygenase 2 (DAO2, At1g14120), and auxin transport proteins PIN2 (At5g57090) and PIN4 (At2g01420). Gene expression was calculated according to Livak [45] and presented as fold change compared to Col-0. Asterisk $\left(^{*}\right)$ indicates significant differences with respect to the Col-0 growth conditions as the control (Student's $t$ test, ${ }^{*}$ presents $p<0.05,{ }^{* *} p<0.01,{ }^{* * *} p<0.001$ ). Bars show means \pm SE of three biological replicates.

\section{Materials and Methods}

\subsection{Reagents and Standards}

Murashige and Skoog (MS) powder medium containing vitamins, plant agar, and 2-(N-morpholino)ethanesulfonic acid (MES) monohydrate were obtained from Duchefa, Haarlem, The Netherlands. Sucrose, $\mathrm{NaCl}$, mannitol were purchased from Kemika, Zagreb, Croatia, while Izosan G was purchased from Pliva, Zagreb, Croatia. Propidium iodide (PI) was obtained from Sigma-Aldrich, St. Louis, MO, USA. Standards for IAA and its precursors anthranilate (ANT), indole-3-acetaldehyde (IAAld), IAM, indole-3-acetonitrile (IAN), IPyA, tryptamine (TRA), and tryptophan (TRP) were purchased from Sigma-Aldrich, and standards for IAOx and 2-oxoindole-3-acetic acid (oxIAA) from OlChemIm Ltd, Olomouc, Czech Republic. Acetic acid was purchased from Merck (Darmstadt, Germany), diethyldithiocarbamic acid sodium salt and cysteamine hydrochloride from Sigma-Aldrich, St. Louis, MO, USA, and HPLC gradient grade solvents from J.T. Baker-Fisher Scientific, Hampton, NH, USA. All other chemicals were from Carl Roth, Karlsruhe, Germany and Sigma-Aldrich. 


\subsection{Plant Material and Growth Conditions}

Seeds of Arabidopsis (Arabidopsis thaliana) ecotype Columbia- 0 and the previously described DR5rev::GFP line (N9361) [56,57] were purchased from The Nottingham Arabidopsis Stock Centre [58]. Seeds of the transgenic Arabidopsis lines pPIN2::PIN2-GFP [59], pPIN4::PIN4-GFP [60], pPIN7::PIN7-GFP [61] were kindly provided by C. Luschnik and J. Friml.

Seeds were surface sterilized with $70 \%$ ethanol $(v / v)$ for $1 \mathrm{~min}$, followed by $1 \%$ Izosan $\mathrm{G}(w / v)$ for $10 \mathrm{~min}$, washed five times with sterile distilled water, seeded on the fullstrength MS medium containing vitamins, $1 \%(w / v)$ plant agar, $1 \%(w / v)$ sucrose, $2,5 \mathrm{mM}$ MES monohydrate, ( $\mathrm{pH}$ 5.7). After stratification for 2 days at $4{ }^{\circ} \mathrm{C}$, plates were transferred to a growth chamber (PHC Corporation, Tokio, Japan), positioned vertically, at $22{ }^{\circ} \mathrm{C}$ under $16 \mathrm{~h}$ of light at $150 \mu \mathrm{mol} \mathrm{m}{ }^{-2} \mathrm{~s}^{-1} / 8 \mathrm{~h}$ darkness. Four-day-old seedlings were used for further experiments.

\subsection{Stress Treatments of Seedlings}

To investigate the effect of the stressors (sodium chloride and mannitol) long-term (up to 13 days) short-term ( 1.5 to $12 \mathrm{~h}$ ) and immediate ( 0 to $15 \mathrm{~min}$ ) analyses of auxin synthesis, accumulation and distribution were performed. For long-term stress application 4-day-old seedlings germinated on MS agar plates supplemented with $2.5 \mathrm{mM}$ MES buffer ( $\mathrm{pH}$ 5.7) were transferred to the fresh plates of the same medium (Control) or to the same medium supplemented with either $100 \mathrm{mM} \mathrm{NaCl}(\mathrm{NaCl})$ or $200 \mathrm{mM}$ mannitol (Man). Seedlings were further cultured for 13 days at $22{ }^{\circ} \mathrm{C}$ under $16 \mathrm{~h}$ of light at $150 \mu \mathrm{mol} \mathrm{m}{ }^{-2}$ $\mathrm{s}^{-1} / 8 \mathrm{~h}$ darkness. For short-term stress application 4-day-old seedlings germinated on MS agar plates supplemented with $2.5 \mathrm{mM}$ MES buffer (pH 5.7) were transferred to $2.5 \mathrm{mM}$ MES buffer (pH 5.7) (Control) or the same buffer supplemented with either $100 \mathrm{mM} \mathrm{NaCl}$ $(\mathrm{NaCl})$ or $200 \mathrm{mM}$ mannitol (Man). For immediate stress application, 4-day-old seedlings germinated on MS agar plates containing $2.5 \mathrm{mM}$ MES buffer (pH 5.7) were transferred to a microscopic slide with a drop of distilled water and observed. Distilled water was replaced by adding an excessive volume of $100 \mathrm{mM} \mathrm{NaCl}$ soaked with filter paper under the coverslip and the seedlings were immediately further analyzed.

For gene expression analysis, the roots of 4-day-old seedlings were directly covered on Petri dishes with $15 \mathrm{~mL}$ of either $2.5 \mathrm{mM}$ MES buffer (pH 5.7) as a control or with $100 \mathrm{mM} \mathrm{NaCl}$ in $2.5 \mathrm{mM}$ MES buffer or $200 \mathrm{mM}$ mannitol in $2.5 \mathrm{mM}$ MES buffer and placed horizontally in the growth chamber for $3 \mathrm{~h}$. After the treatments, roots were excised, frozen with liquid nitrogen, and stored at $-80{ }^{\circ} \mathrm{C}$ until RNA isolation.

\subsection{Root Growth Bioassay}

To investigate the effect of stressors on the root growth rate and the root architecture long-term stress was applied. Root growth bioassay was performed on Arabidopsis ecotype Columbia-0 (Col-0) and the DR5rev::GFP (DR5) lines. Four-day-old seedlings were transferred to media containing stressors or control medium and were grown for the next 13 days on vertically positioned plates in the growth chamber under previously described conditions, and periodically observed for root growth and architecture. Plates were then scanned byHP Scanjet G3010 scanner (Hewlett-Packard, Palo Alto, CA, USA) and root images analyzed using the EZ Rhizo II program (v2.5.0.4, University of Glasgow, Glasgow, UK) [62].

\subsection{Confocal Laser Scanning Microscopy and Image Analyses}

Auxin induced GFP synthesis was followed by confocal microscopy in root cells of 4-day-old DR5 seedlings that were exposed to short-term stress treatment. Seedlings were exposed to the stressors for 3 and $6 \mathrm{~h}$. Based on the preliminary results showing a drastic redistribution of auxin upon short-term salinity stress $(3 \mathrm{~h})$, we further investigated localizations of PINs (PIN2, PIN4, and PIN7) upon immediate salinity stress (5, 10, or $15 \mathrm{~min})$. 
After short-stress treatments, DR5 seedlings were stained in $50 \mu \mathrm{gmL}^{-1}$ propidium iodide (PI) solution for $1 \mathrm{~min}$, rinsed in distilled water, and mounted in water on microscope slides. Images were acquired in 8-bit format using a Leica TCS SP8 X laser scanning confocal microscope with $40 \times$ numerical aperture $(\mathrm{NA})=1.3$ oil-immersion objective and a pinhole corresponding to $1.0 \times$ the diameter of Airy disk or with Leica TCS SP2 laser scanning confocal microscope with $20 \times$ numerical aperture $(\mathrm{NA})=0.70$ and $63 \times \mathrm{NA}=1.40$ oil-immersion objective. The system was controlled with the Leica Application Suite $X$ Software (v3.1.1.15751, Leica Microsystems, Wetzlar, Germany) or with Leica Confocal Software LCS (v2.6.0, Leica Microsystems, Wetzlar, Germany). GFP and PI were excited at $488 \mathrm{~nm}$ by a visible gas Argon laser. GFP emission was detected at 500-530 nm (or $500-563 \mathrm{~nm}$ ), while PI was detected at 597-670 nm. All confocal images were acquired with a constant set of microscopy, detection, and excitation parameters. At least ten independent seedlings were analyzed per treatment, and treatment representative images were selected for figure construction. For the acquisition of a Z-stack image sequence, images were acquired in 20 focal planes with $0.5 \mu \mathrm{m}$ z-spacing, $30 \mathrm{~nm}$ xy-pixel size, and $400 \mathrm{~Hz}$ unidirectional xyz scan mode. For the measurement of fluorescence intensity, optical sections at the focal plane of the quiescent center cells were acquired. Fluorescence intensity was quantified according to Gu et al. [63] using the program Fiji-ImageJ (v1.51n Java 1.8.0_66, National Institutes of Health, Bethesda, MD, USA) [64]. Ten roots per treatment were examined, and at least three independent experiments were performed.

\subsection{Auxin Metabolite Analysis}

Auxin metabolome was assessed in young 4-day-old seedlings of Col-0 and the DR5 line treated with either $100 \mathrm{mM} \mathrm{NaCl}$ or $200 \mathrm{mM}$ mannitol in $2.5 \mathrm{mM}$ MES buffer (pH 5.7) for $3 \mathrm{~h}$. Untreated seedlings grown on $2.5 \mathrm{mM}$ MES buffer ( $\mathrm{pH}$ 5.7) were used as controls. Furthermore, the roots of Arabidopsis plants were also analyzed after prolonged stress conditions (13-day treatments). After the long-term treatment, four to five biological replicates of plant samples were collected and weighed up to $10 \mathrm{mg}$ fresh weight per replicate. Complete auxin profiling was performed using the ultra-rapid auxin metabolite profiling method, previously described [65]. All purified samples were analyzed by a 1260 Infinity LC system combined with a 6495 Triple Quadrupole LC/MS system (Agilent Technologies, Santa Clara, CA, USA) using stable isotope-labeled internal standards as a reference.

\subsection{Real-Time PCR of Selected Genes}

Each sample (biological replicate) for RNA isolation contained control or stress treated roots from 75 seedlings that were frozen in liquid nitrogen and stored at $-80^{\circ} \mathrm{C}$. For total RNA isolation, $500 \mu \mathrm{L}$ of RNAzol (Sigma Aldrich, St. Louis, MO, USA) was added to the sample, after which the roots were homogenized on the Mixer Mill MM400 (Retsch $\mathrm{GmbH}$, Haan, Germany) in three cycles of $1 \mathrm{~min}$ at $30 \mathrm{~Hz}$ with zirconium oxide beads (Next Advance, Troy, NY, USA). After centrifugation, an equal volume of ethanol (100\%) was added to the supernatants, which were further processed using the Direct-zol ${ }^{\mathrm{TM}}$ RNA MiniPrep kit (Zymo Research, Irvine, CA, USA) according to the manufacturer's instructions. DNA was removed with recombinant RNase-free Dnase I (Roche Diagnostics GmbH, MannheimGermany). Up to $30 \mu \mathrm{L}$ of total RNA $(1 \mu \mathrm{g})$ was incubated with $1 \mathrm{U}$ of DNase for $30 \mathrm{~min}$ at $37^{\circ} \mathrm{C}$ in $1 \mathrm{X}$ incubation buffer, followed by the addition of $250 \mu \mathrm{L}$ of Binding Buffer from GeneJET RNA Cleanup and Concentration Micro Kit (ThermoScientific, Waltham, MA, USA) and subsequent purification according to the manufacturer's instruction. The final concentrated DNase treated RNA was eluted in $10 \mu \mathrm{L}$ of RNase free water. RNA integrity was checked before and after DNase I treatment by measuring the absorbance with NanoDrop ND-2000 spectrophotometer (NanoDrop Technologies, Wilmington, DE, USA) and by non-denaturing agarose gel-electrophoresis. cDNA was synthesized using Maxima First Strand cDNA Synthesis Kit for qPCR (ThermoScientific, Waltham, MA, USA) according to the manufacturer's protocol. Reverse transcription was performed with $0.3 \mu \mathrm{g}$ 
of DNase treated total RNA in a thermocycler (Applied Biosystems, Waltham, MA, USA) under the following conditions: $10 \mathrm{~min}$ at $25^{\circ} \mathrm{C}$, followed by $15 \mathrm{~min}$ at $50{ }^{\circ} \mathrm{C}$ and $5 \mathrm{~min}$ at $85^{\circ} \mathrm{C}$.

Gene-specific primers were designed using Quant Prime [66] and Primer-BLAST [67] and the reference gene was selected according to Czechowski et al. [68]. All the primers used are listed in Supplemental Material Table S2. Quantitative PCR was performed in a 96-well optical plate using the 7300 Real-Time PCR system (Applied Biosystems) and universal cycling conditions $\left(10 \mathrm{~min} 95^{\circ} \mathrm{C}, 40\right.$ cycles of $15 \mathrm{~s}$ at $95{ }^{\circ} \mathrm{C}$ and $60 \mathrm{~s}$ at $60{ }^{\circ} \mathrm{C}$ ) followed by the generation of a dissociation curve to check for the specificity of the amplification. Reactions contained Power SYBR Green Master Mix (Applied Biosystems), $200 \mathrm{nM}$ of a gene-specific forward and reverse primer, and $3 \mu \mathrm{L}$ of 10x diluted cDNA in each $15 \mu \mathrm{L}$ reaction. No template controls (NTC) contained $3 \mu \mathrm{L}$ RNase free water instead. For qPCR, three biological replicates were performed in triplicate (technical replicates). PCR amplification efficiencies were calculated using LinRegPCR software v2020.2 [69]. Supplemental Material Table S2 shows the amplification efficiencies for the primer pairs we studied. Relative expression (fold change) was calculated using the comparative $2^{-\Delta \Delta \mathrm{Ct}}$ method [45] with the TIP41- like gene (AT4G34270) as the reference gene. Statistical analysis of the obtained data was performed using Student's $t$-test to detect the differences between control and treated groups. The results are presented as mean $\pm \mathrm{SE}$ of three independent experiments. The standard error was calculated using the results of the $2^{-\Delta \Delta \mathrm{Ct}}$ method. The chromosomal locations for the studied genes have been depicted based on data from TAIR10 assembly by Chromosome viewer at bar.utoronto.ca/eplant [70].

\subsection{Statistical Analysis}

Significance of differences was determined using Student's test, as indicated in figures ${ }^{*},{ }^{* *}$, and ${ }^{* *}$ correspond to $p$-values of $0.05>p>0.01,0.01>p>0.001$, and $p<0.001$, respectively). Each data point indicated represents the average of three replicates $(n=3)$ unless otherwise stated.

\section{Conclusions}

We examined the effect of short-term $(3 \mathrm{~h})$ and prolonged (13 days) salt $(100 \mathrm{mM}$ $\mathrm{NaCl}$ ) and osmotic (200 mM mannitol) stress on the auxin metabolome, auxin distribution, and transcript levels of selected auxin related genes in Arabidopsis Col-0 and DR5 line. Both abiotic stresses caused inhibition of main root growth and lateral root development in response to treatment duration, with DR5 lines being more affected compared to Col- 0 . Prolonged osmotic stress caused more drastic changes in root growth and LRs development compared with salt stress, probably due to the ability of plants to neutralize $\mathrm{NaCl}$ by compartmentalization. Short-term $(3 \mathrm{~h})$ stress caused significant changes in the auxin metabolome, especially the $\mathrm{NaCl}$ treatment, which resulted in a significant IAA decrease compared with the control. This is in agreement with the auxin distribution observed by confocal microscopy in the DR5 line. $\mathrm{NaCl}$ treatment resulted in a redistribution of auxin signaling from the quiescent center and the internal layers of the root cap (common to control and mannitol-treated roots) to the epidermal and cortical cells of the root elongation zone. PIN protein distribution and gene expression were also affected by stress conditions. PINs, especially PIN2 protein concentration were reduced during the first 5 min of salt treatment. This observation was also consistent with the auxin related transcriptome data from Col-0, suggesting disruption of auxin biosynthesis genes expression, but particularly amide and ester conjugation processes. Indeed, analysis of the transcription level of selected genes involved in auxin metabolism mainly confirmed higher transcription of YUC, GH3s, and UDP genes in both lines, whereas transcription of PINs was lower under stress conditions. Certain differences were observed between the Col-0 and DR5 lines, which showed a lower transcription level of YUC5 and PINs. In contrast, the transcription level of GH3 genes and DAO2 was higher in the DR5 line than in the Col-0. Moreover, our gene expression data correspond to the differences in the auxin metabolome determined 
between the two lines. Interestingly, Col-0 seedlings contained more IAA, whereas DR5 seedlings had higher levels of the amide conjugates IAA-Glu and IAA-Asp. During prolonged stress conditions, the auxin metabolome showed an intention to stabilize, and a tendency to increase IAA was observed, especially under salt stress.

In conclusion, salinity stress, a complex stress composed of the toxic and osmotic effects of salt, causes more striking changes in the distribution of auxin as well as in its metabolome and transcriptome during a short treatment. However, a longer stress treatment with $\mathrm{NaCl}$ caused less conspicuous changes in root growth and architecture, while IAA content increased more frequently. Based on the results under our experimental conditions, the DR5 line, which is commonly used for auxin monitoring, appeared to be more sensitive to the applied stresses than the wild type Col-0, although the trends in morphological, metabolome, and transcriptome parameters are similar. To our opinion, this observation is useful and new information that may help researchers who use the DR5 auxin reporter line in explanations of maybe unexpected results. As far as adequate control is included there is no doubt that this line is a very useful and helpful research tool in the visualization of auxin distribution.

Supplementary Materials: The following are available online at https:/ /www.mdpi.com/article/10 .3390/ijms22157993/s1, Figure S1: Seedlings morphology of Arabidopsis thaliana Columbia-0 (Col 0-WT) and DR5rev::GFP line (DR5) grown on MS medium (control) or treated with $100 \mathrm{mM} \mathrm{NaCl}$ $(\mathrm{NaCl})$ or $200 \mathrm{mM}$ mannitol (Man) for 13 days. Figure S2: PIN2 proteins distribution in the root tips of Arabidopsis pPIN2::PIN2-GFP line in response to salt stress $(100 \mathrm{mM} \mathrm{NaCl})$ and corresponding controls in time range 5-15 min. Figure S3: PIN4 proteins distribution in the root tips of Arabidopsis pPIN4::PIN42-GFP line in response to salt stress $(100 \mathrm{mM} \mathrm{NaCl})$ and corresponding controls in time range 5-15 min. Figure S4: PIN7 proteins distribution in the root tips of Arabidopsis pPIN74::PIN47GFP line in response to salt stress $(100 \mathrm{mM} \mathrm{NaCl})$ and corresponding controls in time range 5-15 $\mathrm{min}$. Figure S5. The chromosomal locations for the genes studied in Figures 9 and 10 based on the genome assembly of Arabidopsis thaliana visualized by Chromosome viewer at bar.utoronto.ca/eplant [70]. Table S1: Micro-array data in salt stress $(150 \mathrm{mM} \mathrm{NaCl})$ and osmotic stress ( $300 \mathrm{mM}$ mannitol) in time range 0.5-24 has been published according to Killian et al. [42] for genes involved in auxin metabolism. The data has been derived from the eFP browser website [43], and the normalization has been used as published on this website. IAA metabolism is illustrated in Figure 1. Relative values represent $\log 2$ fold change of expression upon salt and mannitol treatments. The expression level is marked by gradation of color intensity: from bright to dark blue (decrease in expression), over white (unaltered expression), from bright to dark red (increase in expression). Table S2: qPCR primer list and corresponding amplification efficiencies as calculated by Ruijter et al. [69]. Video S1: Z-stack of DR5rev::GFP mock treated root made by laser scanning confocal microscopy as described in Materials and Methods. A stack of 20 images ( $0.5 \mu \mathrm{m}$ spacing) of optical sections around the quiescent center of roots mock treated for $3 \mathrm{~h}$ (in 1xMS, $2.5 \mathrm{mM}$ MES, pH 5.7 supplemented with water as mock treatment). Z-stack was depicted in ImageJ as a video of a combined stack of three channels showed from left to right as GFP channel (GFP signal transformed with LUT Fire option), bright field channel (middle), and merged channel (furthest right). Scale bar- $50 \mu \mathrm{m}$, calibration bar-range of pixel intensities from 0-255. Video S2: Z-stack of DR5rev::GFP mannitol treated root made by laser scanning confocal microscopy as described in Materials and Methods. A stack of 20 images ( $0.5 \mu \mathrm{m}$ spacing) of optical sections around the quiescent center of roots treated with mannitol for $3 \mathrm{~h}$ (in 1xMS, $2.5 \mathrm{mM}$ MES, pH 5.7 supplemented with $200 \mathrm{mM}$ mannitol). Z-stack was depicted in ImageJ as a video of a combined stack of three channels showed from left to right as GFP channel (GFP signal transformed with LUT Fire option), brightfield channel (middle), and merged channel (furthest right). Scale bar- $50 \mu \mathrm{m}$, calibration bar-range of pixel intensities from 0-255. Video S3: Z-stack of DR5rev::GFP salt treated root made by laser scanning confocal microscopy as described in Materials and Methods. A stack of 20 images $(0.5 \mu \mathrm{m}$ spacing) of optical sections around the quiescent center of roots treated with $\mathrm{NaCl}$ for $3 \mathrm{~h}$ (in 1xMS, $2.5 \mathrm{mM}$ MES, pH 5.7 supplemented with either $100 \mathrm{mM} \mathrm{NaCl}$ ). Z-stack was depicted in ImageJ as a video of a combined stack of three channels showed from left to right as GFP channel (GFP signal transformed with LUT Fire option), bright field channel (middle), and merged channel (furthest right). Scale bar- $50 \mu \mathrm{m}$, calibration bar-range of pixel intensities from 0-255. 
Author Contributions: Conceptualization, B.S.-S., A.S. and N.B.; methodology, A.S., N.B. and I.P.; investigation, A.S., N.B., I.P. and B.S.-S.; writing-original draft preparation, B.S.-S.; writing-review and editing, A.S., N.B., I.P., A.P., O.N. and B.S.-S.; visualization, A.S., N.B. and B.S.-S.; supervision, A.P. and O.N.; project administration, B.S.-S.; funding acquisition, B.S.-S. and O.N. All authors have read and agreed to the published version of the manuscript.

Funding: This research was funded by the Croatian Science Foundation (Project No. IP-2014-094359), by the Operational Programme Competitiveness and Cohesion 2014-2020 and by the Croatian European regional fund under a specific scheme to strengthen applied research in proposing actions for climate change adaptation (Project No. KK.05.1.1.02.0005), by the Ministry of Education, Youth and Sports of the Czech Republic (European Regional Development Fund-Project "Plants as a tool for sustainable global development" No. CZ.02.1.01/0.0/0.0/16_019/0000827), and by the Palacký University Olomouc Young Researcher grant (No. JG_2020_002).

Institutional Review Board Statement: Not applicable.

Informed Consent Statement: Not applicable.

Acknowledgments: The authors would like to thank Karel Doležal for critical reading and discussions and Lucija Horvat for technical support and expertise in the operation of the Leica TCS SP8 X laser scanning confocal microscope and the Leica TCS SP2 laser scanning confocal microscope.

Conflicts of Interest: The authors declare no conflict of interest.

\section{References}

1. Daliakopoulos, I.N.; Panagea, I.S.; Tsanis, I.K.; Grillakis, M.G.; Koutroulis, A.G.; Hessel, R.; Mayor, A.G.; Ritsema, C.J. Yield response of Mediterranean rangelands under a changing climate. Land Degrad. Dev. 2017, 28, 1962-1972. [CrossRef]

2. Petersson, S.V.; Johansson, A.I.; Kowalczyk, M.; Makoveychuk, A.; Wang, J.Y.; Moritz, T.; Grebe, M.; Benfey, P.N.; Sandberg, G.; Ljung, K. An auxin gradient and maximum in the Arabidopsis root apex shown by high-resolution cell-specific analysis of IAA distribution and synthesis. Plant Cell 2009, 21, 1659-1668. [CrossRef] [PubMed]

3. Clark, N.M.; de Luis Balaguer, M.A.; Sozzani, R. Experimental data and computational modeling link auxin gradient and development in the Arabidopsis root. Front. Plant Sci. 2014, 5, 328. [CrossRef] [PubMed]

4. Korver, R.A.; Koevoets, I.T.; Testerink, C. Out of shape during stress: A key role for auxin. Trends Plant Sci. $2018,23,783-793$. [CrossRef]

5. $\quad$ Ljung, K.; Hull, A.K.; Celenza, J.; Yamada, M.; Estelle, M.; Normanly, J.; Sandberg, G. Sites and regulation of auxin biosynthesis in Arabidopsis roots. Plant Cell 2005, 17, 1090-1104. [CrossRef]

6. Du, Y.; Scheres, B. Lateral root formation and the multiple roles of auxin. J. Exp. Bot. 2018, 69, 155-167. [CrossRef]

7. Tivendale, N.D.; Ross, J.J.; Cohen, J.D. The shifting paradigms of auxin biosynthesis. Trends Plant Sci. 2014, 19, 44-51. [CrossRef]

8. Di, D.-W.; Zhang, C.; Luo, P.; An, C.-W.; Guo, G.-Q. The biosynthesis of auxin: How many paths truly lead to IAA? Plant Growth Regul. 2016, 78, 275-285. [CrossRef]

9. Olatunji, D.; Geelen, D.; Verstraeten, I. Control of endogenous auxin levels in plant root development. Int. J. Mol. Sci. 2017, 18, 2587. [CrossRef]

10. Zhang, J.; Peer, W.A. Auxin homeostasis: The DAO of catabolism. J. Exp. Bot. 2017, 68, 3145-3154. [CrossRef] [PubMed]

11. Smolko, A.; Ludwig-Müller, J.; Salopek-Sondi, B. Auxin Amidohydrolases-From Structure to Function: Revisited. Croat. Chem. Acta 2018, 91, 233-239. [CrossRef]

12. Casanova-Sáez, R.; Mateo-Bonmatí, E.; Ljung, K. Auxin metabolism in plants. Cold Spring Harb. Perspect. Biol. 2021, 13 , a039867. [CrossRef] [PubMed]

13. Kanehisa, M. KEGG: Kyoto Encyclopedia of Genes and Genomes. Nucleic Acids Res. 2000, 28, 27-30. [CrossRef]

14. Normanly, J.; Slovin, J.P.; Cohen, J.D. Auxin biosynthesis and metabolism. In Plant Hormones; Davies, P.J., Ed.; Springer: Dordrecht, The Netherlands, 2010; pp. 36-62.

15. Ludwig-Müller, J. Auxin conjugates: Their role for plant development and in the evolution of land plants. J. Exp. Bot. 2011, 62, 1757-1773. [CrossRef]

16. Bartel, B.; LeClere, S.; Magidin, M.; Zolman, B.K. Inputs to the active indole-3-acetic acid pool: De novo synthesis, conjugate hydrolysis, and indole-3-butyric acid $\beta$-oxidation. J. Plant Growth Regul. 2001, 20, 198-216. [CrossRef]

17. Sánchez-García, A.B.; Ibáñez, S.; Cano, A.; Acosta, M.; Pérez-Pérez, J.M. A comprehensive phylogeny of auxin homeostasis genes involved in adventitious root formation in carnation stem cuttings. PLoS ONE 2018, 13, e0196663. [CrossRef]

18. Korasick, D.A.; Enders, T.A.; Strader, L.C. Auxin biosynthesis and storage forms. J. Exp. Bot. 2013, 64, 2541-2555. [CrossRef]

19. Tanaka, K.; Hayashi, K.; Natsume, M.; Kamiya, Y.; Sakakibara, H.; Kawaide, H.; Kasahara, H. UGT74D1 Catalyzes the Glucosylation of 2-Oxindole-3-Acetic Acid in the Auxin Metabolic Pathway in Arabidopsis. Plant Cell Physiol. 2014, 55, 218-228. [CrossRef] [PubMed] 
20. Sugawara, S.; Hishiyama, S.; Jikumaru, Y.; Hanada, A.; Nishimura, T.; Koshiba, T.; Zhao, Y.; Kamiya, Y.; Kasahara, H. Biochemical analyses of indole-3-acetaldoxime-dependent auxin biosynthesis in Arabidopsis. Proc. Natl. Acad. Sci. USA 2009, 106, $5430-5435$. [CrossRef]

21. Tzin, V.; Galili, G. The Biosynthetic Pathways for Shikimate and Aromatic Amino Acids in Arabidopsis thaliana. Arabidopsis Book 2010, 8, e0132. [CrossRef] [PubMed]

22. Abualia, R.; Benkova, E.; Lacombe, B. Transporters and Mechanisms of Hormone Transport in Arabidopsis. In Membrane Transport in Plants; Maurel, C.B., Ed.; Academic Press: Cambridge, MA, USA, 2018; Volume 87, pp. 115-138. [CrossRef]

23. Mohanta, T.K.; Bashir, T.; Hashem, A.; Abd_Allah, E.F.; Khan, A.L.; Al-Harrasi, A.S. Molecular players of auxin transport systems: Advances in genomic and molecular events. J. Plant Interact. 2018, 13, 483-495. [CrossRef]

24. Koevoets, I.T.; Venema, J.H.; Elzenga, J.T.M.; Testerink, C. Roots Withstanding their Environment: Exploiting Root System Architecture Responses to Abiotic Stress to Improve Crop Tolerance. Front. Plant Sci. 2016, 7, 1335. [CrossRef]

25. Mroue, S.; Simeunovic, A.; Robert, H.S. Auxin production as an integrator of environmental cues for developmental growth regulation. J. Exp. Bot. 2018, 69, 201-212. [CrossRef]

26. Liang, W.; Ma, X.; Wan, P.; Liu, L. Plant salt-tolerance mechanism: A review. Biochem. Biophys. Res. Commun. 2018, 495, 286-291. [CrossRef]

27. Salopek-Sondi, B.; Pavlović, I.; Smolko, A.; Šamec, D. Auxin as a Mediator of Abiotic Stress Responses. In Mechanism of Plant Hormone Signaling under Stress; Pandey, G., Ed.; John Wiley \& Sons, Ltd.: Hoboken, NJ, USA, 2017; pp. 1-36.

28. Wang, Y.; Li, K.; Li, X. Auxin redistribution modulates plastic development of root system architecture under salt stress in Arabidopsis thaliana. J. Plant Physiol. 2009, 166, 1637-1645. [CrossRef] [PubMed]

29. Julkowska, M.M.; Hoefsloot, H.C.J.; Mol, S.; Feron, R.; de Boer, G.-J.; Haring, M.A.; Testerink, C. Capturing Arabidopsis Root Architecture Dynamics with ROOT-FIT Reveals Diversity in Responses to Salinity. Plant Physiol. 2014, 166, 1387-1402. [CrossRef]

30. Ryu, H.; Cho, Y.-G. Plant hormones in salt stress tolerance. J. Plant Biol. 2015, 58, 147-155. [CrossRef]

31. Pavlović, I.; Pěnčík, A.; Novák, O.; Vujčić, V.; Radić Brkanac, S.; Lepeduš, H.; Strnad, M.; Salopek-Sondi, B. Short-term salt stress in Brassica rapa seedlings causes alterations in auxin metabolism. Plant Physiol. Biochem. 2018, 125, 74-84. [CrossRef]

32. Julkowska, M.M.; Koevoets, I.T.; Mol, S.; Hoefsloot, H.; Feron, R.; Tester, M.A.; Keurentjes, J.J.B.; Korte, A.; Haring, M.A.; de Boer, G.-J.; et al. Genetic Components of Root Architecture Remodeling in Response to Salt Stress. Plant Cell 2017, 29, 3198-3213. [CrossRef] [PubMed]

33. Galvan-Ampudia, C.S.; Testerink, C. Salt stress signals shape the plant root. Curr. Opin. Plant Biol. 2011, 14, 296-302. [CrossRef]

34. Liu, W.; Li, R.-J.; Han, T.-T.; Cai, W.; Fu, Z.-W.; Lu, Y.-T. Salt Stress Reduces Root Meristem Size by Nitric Oxide-Mediated Modulation of Auxin Accumulation and Signaling in Arabidopsis. Plant Physiol. 2015, 168, 343-356. [CrossRef]

35. Zhang, J.; Lin, J.E.; Harris, C.; Campos Mastrotti Pereira, F.; Wu, F.; Blakeslee, J.J.; Peer, W.A. DAO1 catalyzes temporal and tissue-specific oxidative inactivation of auxin in Arabidopsis thaliana. Proc. Natl. Acad. Sci. USA 2016, 113, 11010-11015. [CrossRef]

36. Liu, L.; Guo, G.; Wang, Z.; Ji, H.; Mu, F.; Li, X. Auxin in Plant Growth and Stress Responses. In Phytohormones: A Window to Metabolism, Signaling and Biotechnological Applications; Phan Tran, L.-S., Pal, S., Eds.; Springer: New York, NY, USA, 2014; pp. 1-35.

37. Šimura, J.; Antoniadi, I.; Široká, J.; Tarkowská, D.; Strnad, M.; Ljung, K.; Novák, O. Plant hormonomics: Multiple phytohormone profiling by targeted metabolomics. Plant Physiol. 2018, 177, 476-489. [CrossRef] [PubMed]

38. Křeček, P.; Skůpa, P.; Libus, J.; Naramoto, S.; Tejos, R.; Friml, J.; Zažímalová, E. The PIN-FORMED (PIN) protein family of auxin transporters. Genome Biol. 2009, 10, 249. [CrossRef] [PubMed]

39. Jiang, K.; Moe-Lange, J.; Hennet, L.; Feldman, L.J. Salt Stress Affects the Redox Status of Arabidopsis Root Meristems. Front. Plant Sci. 2016, 7, 81. [CrossRef]

40. Feraru, E.; Friml, J. PIN Polar Targeting. Plant Physiol. 2008, 147, 1553-1559. [CrossRef]

41. Fu, Y.; Yang, Y.; Chen, S.; Ning, N.; Hu, H. Arabidopsis IAR4 Modulates Primary Root Growth Under Salt Stress Through ROS-Mediated Modulation of Auxin Distribution. Front. Plant Sci. 2019, 10, 522. [CrossRef]

42. Kilian, J.; Whitehead, D.; Horak, J.; Wanke, D.; Weinl, S.; Batistic, O.; D'Angelo, C.; Bornberg-Bauer, E.; Kudla, J.; Harter, K. The AtGenExpress global stress expression data set: Protocols, evaluation and model data analysis of UV-B light, drought and cold stress responses. Plant J. 2007, 50, 347-363. [CrossRef] [PubMed]

43. Winter, D.; Vinegar, B.; Nahal, H.; Ammar, R.; Wilson, G.V.; Provart, N.J. An “Electronic Fluorescent Pictograph" Browser for Exploring and Analyzing Large-Scale Biological Data Sets. PLoS ONE 2007, 2, e718. [CrossRef]

44. Boyes, D.C.; Zayed, A.M.; Ascenzi, R.; McCaskill, A.J.; Hoffman, N.E.; Davis, K.R.; Görlach, J. Growth stage-based phenotypic analysis of Arabidopsis: A model for high throughput functional genomics in plants. Plant Cell 2001, 13, 1499-1510. [CrossRef] [PubMed]

45. Livak, K.J.; Schmittgen, T.D. Analysis of Relative Gene Expression Data Using Real-Time Quantitative PCR and the $2-\Delta \Delta C T$ Method. Methods 2001, 25, 402-408. [CrossRef] [PubMed]

46. Yan, S.; Che, G.; Ding, L.; Chen, Z.; Liu, X.; Wang, H.; Zhao, W.; Ning, K.; Zhao, J.; Tesfamichael, K.; et al. Different cucumber CsYUC genes regulate response to abiotic stresses and flower development. Sci. Rep. 2016, 6, 20760. [CrossRef]

47. Shi, H.; Chen, L.; Ye, T.; Liu, X.; Ding, K.; Chan, Z. Modulation of auxin content in Arabidopsis confers improved drought stress resistance. Plant Physiol. Biochem. 2014, 82, 209-217. [CrossRef] 
48. Kim, J.I.; Baek, D.; Park, H.C.; Chun, H.J.; Oh, D.-H.; Lee, M.K.; Cha, J.-Y.; Kim, W.-Y.; Kim, M.C.; Chung, W.S.; et al. Overexpression of Arabidopsis YUCCA6 in potato results in high-auxin developmental phenotypes and enhanced resistance to water deficit. Mol. Plant 2013, 6, 337-349. [CrossRef] [PubMed]

49. Ke, Q.; Wang, Z.; Ji, C.Y.; Jeong, J.C.; Lee, H.-S.; Li, H.; Xu, B.; Deng, X.; Kwak, S.-S. Transgenic poplar expressing Arabidopsis YUCCA6 exhibits auxin-overproduction phenotypes and increased tolerance to abiotic stress. Plant Physiol. Biochem. 2015, 94, 19-27. [CrossRef] [PubMed]

50. Park, J.-E.; Park, J.-Y.; Kim, Y.-S.; Staswick, P.E.; Jeon, J.; Yun, J.; Kim, S.-Y.; Kim, J.; Lee, Y.-H.; Park, C.-M. GH3-mediated auxin homeostasis links growth regulation with stress adaptation response in Arabidopsis. J. Biol. Chem. 2007, 282, 10036-10046. [CrossRef] [PubMed]

51. Kirungu, J.N.; Magwanga, R.O.; Lu, P.; Cai, X.; Zhou, Z.; Wang, X.; Peng, R.; Wang, K.; Liu, F. Functional characterization of Gh_A08G1120 (GH3.5) gene reveal their significant role in enhancing drought and salt stress tolerance in cotton. BMC Genet. 2019, 20, 62. [CrossRef] [PubMed]

52. Kong, W.; Zhong, H.; Deng, X.; Gautam, M.; Gong, Z.; Zhang, Y.; Zhao, G.; Liu, C.; Li, Y. Evolutionary analysis of GH3 genes in six Oryza species/subspecies and their expression under salinity stress in Oryza sativa ssp. japonica. Plants 2019, 8, 30. [CrossRef]

53. Kong, W.; Zhang, Y.; Deng, X.; Li, S.; Zhang, C.; Li, Y. Comparative genomic and transcriptomic analysis suggests the evolutionary dynamic of GH3 genes in Gramineae crops. Front. Plant Sci. 2019, 10, 1297. [CrossRef]

54. Kinoshita, N.; Wang, H.; Kasahara, H.; Liu, J.; MacPherson, C.; Machida, Y.; Kamiya, Y.; Hannah, M.A.; Chua, N.-H. IAA-Ala Resistant3, an evolutionarily conserved target of miR167, mediates Arabidopsis root architecture changes during high osmotic stress. Plant Cell 2012, 24, 3590-3602. [CrossRef]

55. Rehman, H.M.; Nawaz, M.A.; Shah, Z.H.; Ludwig-Müller, J.; Chung, G.; Ahmad, M.Q.; Yang, S.H.; Lee, S.I. Comparative genomic and transcriptomic analyses of Family-1 UDP glycosyltransferase in three Brassica species and Arabidopsis indicates stress-responsive regulation. Sci. Rep. 2018, 8, 1875. [CrossRef] [PubMed]

56. Benková, E.; Michniewicz, M.; Sauer, M.; Teichmann, T.; Seifertová, D.; Jürgens, G.; Friml, J. Local, efflux-dependent auxin gradients as a common module for plant organ formation. Cell 2003, 115, 591-602. [CrossRef]

57. Friml, J.; Vieten, A.; Sauer, M.; Weijers, D.; Schwarz, H.; Hamann, T.; Offringa, R.; Jürgens, G. Efflux-dependent auxin gradients establish the apical-basal axis of Arabidopsis. Nature 2003, 426, 147-153. [CrossRef] [PubMed]

58. Scholl, R.L.; May, S.T.; Ware, D.H. Seed and Molecular Resources for Arabidopsis. Plant Physiol. 2000, 124, 1477-1480. [CrossRef] [PubMed]

59. Abas, L.; Benjamins, R.; Malenica, N.; Paciorek, T.; Wišniewska, J.; Moulinier-Anzola, J.C.; Sieberer, T.; Friml, J.; Luschnig, C. Intracellular trafficking and proteolysis of the Arabidopsis auxin-efflux facilitator PIN2 are involved in root gravitropism. Nat. Cell Biol. 2006, 8, 249-256. [CrossRef] [PubMed]

60. Vieten, A.; Vanneste, S.; Wisniewska, J.; Benkova, E.; Benjamins, R.; Beeckman, T.; Luschnig, C.; Friml, J. Functional redundancy of PIN proteins is accompanied by auxin-dependentcross-regulation of PIN expression. Development 2005, 132, 4521-4531. [CrossRef]

61. Blilou, I.; Xu, J.; Wildwater, M.; Willemsen, V.; Paponov, I.; Friml, J.; Heidstra, R.; Aida, M.; Palme, K.; Scheres, B. The PIN auxin efflux facilitator network controls growth and patterning in Arabidopsis roots. Nature 2005, 433, 39-44. [CrossRef] [PubMed]

62. Shahzad, Z.; Kellermeier, F.; Armstrong, E.M.; Rogers, S.; Lobet, G.; Amtmann, A.; Hills, A. EZ-Root-VIS: A software pipeline for the rapid analysis and visual reconstruction of root system architecture. Plant Physiol. 2018, 177, 1368-1381. [CrossRef]

63. Gu, X.; Xu, T.; He, Y. A Histone H3 Lysine-27 Methyltransferase complex represses lateral root formation in Arabidopsis thaliana. Mol. Plant 2014, 7, 977-988. [CrossRef]

64. Schindelin, J.; Arganda-Carreras, I.; Frise, E.; Kaynig, V.; Longair, M.; Pietzsch, T.; Preibisch, S.; Rueden, C.; Saalfeld, S.; Schmid, B.; et al. Fiji: An open-source platform for biological-image analysis. Nat. Methods 2012, 9, 676-682. [CrossRef] [PubMed]

65. Pěnčík, A.; Casanova-Sáez, R.; Pilařová, V.; Žukauskaitė, A.; Pinto, R.; Micol, J.L.; Ljung, K.; Novák, O. Ultra-rapid auxin metabolite profiling for high-throughput mutant screening in Arabidopsis. J. Exp. Bot. 2018, 69, 2569-2579. [CrossRef] [PubMed]

66. Arvidsson, S.; Kwasniewski, M.; Riano-Pachon, D.M.; Mueller-Roeber, B. QuantPrime-A flexible tool for reliable highthroughput primer design for quantitative PCR. BMC Bioinform. 2008, 9, 465. [CrossRef] [PubMed]

67. Ye, J.; Coulouris, G.; Zaretskaya, I.; Cutcutache, I.; Rozen, S.; Madden, T.L. Primer-BLAST: A tool to design target-specific primers for polymerase chain reaction. BMC Bioinform. 2012, 13, 134. [CrossRef] [PubMed]

68. Czechowski, T.; Stitt, M.; Altmann, T.; Udvardi, M.K.; Scheible, W.-R. Genome-wide identification and testing of superior reference genes for transcript normalization in Arabidopsis. Plant Physiol. 2005, 139, 5-17. [CrossRef]

69. Ruijter, J.M.; Ramakers, C.; Hoogaars, W.M.H.; Karlen, Y.; Bakker, O.; van den Hoff, M.J.B.; Moorman, A.F.M. Amplification efficiency: Linking baseline and bias in the analysis of quantitative PCR data. Nucleic Acids Res. 2009, 37, e45. [CrossRef] [PubMed]

70. Waese, J.; Fan, J.; Pasha, A.; Yu, H.; Fucile, G.; Shi, R.; Cumming, M.; Kelley, L.A.; Sternberg, M.J.; Krishnakumar, V.; et al. ePlant: Visualizing and exploring multiple levels of data for hypothesis generation in plant biology. Plant Cell 2017, 29, $1806-1821$. [CrossRef] 